"Shareholder Heterogeneity, Asymmetric Information, and the Equilibrium Manager"

Milo Bianchi, Rose-Anne Dana, Elyès Jouini 


\title{
Shareholder Heterogeneity, Asymmetric Information, and the Equilibrium Manager*
}

\author{
Milo Bianchi ${ }^{\dagger} \quad$ Rose-Anne Dana ${ }^{\ddagger} \quad$ Elyès Jouini $^{\S}$
}

January 2021

Forthcoming in Economic Theory

\begin{abstract}
Consider a firm owned by shareholders with heterogeneous beliefs and discount rates who delegate to a manager the choice of a production plan. The shareholders and the manager can trade contingent claims in a complete asset market. Shareholders cannot observe the chosen production plan and design a compensation scheme so that at equilibrium the manager chooses the plan they prefer and reveals it truthfully. We show that at equilibrium i) profit is maximized, ii) the manager gets a constant share of production, iii) she has no incentive to trade. We then show that such equilibrium exists if and only if the manager has the same belief and discount rate as the representative shareholder. This allows us to characterize the required characteristics of the manager as a function of shareholders' characteristics.
\end{abstract}

${ }^{*}$ We thank three anonymous referees and seminar participants at Columbia, Lausanne, D-TEA Paris, Toulouse for very useful discussions. Milo Bianchi acknowledges funding from ANR (ANR-17-EURE-0010 grant). We have no material interest that relates to the research described in the paper.

†Toulouse School of Economics, TSM, and IUF, University of Toulouse Capitole, Toulouse, France. E-mail: milo.bianchi@tse-fr.eu

${ }^{\ddagger}$ Université Paris-Dauphine, PSL and IPAG Business School, dana@ceremade.dauphine.fr

$\S$ Université Paris-Dauphine, PSL, jouini@ceremade.dauphine.fr 
JEL codes: G32, G34, D24, D51, D53, D70

Keywords: heterogeneous shareholders, asymmetric information, manager-shareholders equilibrium. 


\section{Introduction}

A fundamental literature dating back at least to Berle and Means (1932) analyzes how managers should be chosen and remunerated so as to act in shareholders' best interest. A first challenge is how to define shareholders' interest, given that corporations are often owned by many investors with possibly different tastes and beliefs. Jensen and Meckling (1976) famously proposed to define a "corporate objective function" as the result of "a complex process in which the conflicting objectives of individuals are brought into equilibrium."

A large part of the following literature has studied agency problems from the perspective of a representative shareholder, abstracting from the underlying equilibrium process. In this paper, we take a different perspective. Assuming that shareholders delegate the production choice to a manager and that they can trade assets with her in a complete financial market, we model explicitly how heterogeneous shareholders may reach an agreement on a commonly preferred production plan. Assuming further that the actions taken by the manager cannot be observed, we investigate how to define the characteristics of the manager and her compensation so as to make her act in shareholders' interest.

In our model, shareholders have heterogeneous discount rates and beliefs. The manager chooses a production plan, which determines a flow of uncertain output over time. Shareholders cannot observe the plan chosen by the manager, they only observe the realized production. A contract for the manager specifies her compensation at each date as a function of the history of production.

Given a compensation scheme and a price process, the manager considers the indirect utility associated to each possible production plan, that is the maximal utility she can obtain by choosing a consumption plan subject to her budget constraint. She then chooses a production plan that maximizes her indirect utility and the associated optimal consumption plan and announces the production plan to shareholders. Each shareholder chooses a consumption plan so as to maximize her expected utility subject to her budget constraint. A manager-shareholders equilibrium is then defined by a compensation scheme, a production plan, a price process and a list of 
optimal consumption plans for the manager and shareholders such that, as usual, markets clear and with the following distinctive features: the manager has no incentive to misreport the chosen production plan and the chosen production plan is unanimously preferred by all shareholders.

We start by deriving some intermediary results that define some properties that our equilibrium must satisfy. First, we show that an equilibrium plan maximizes the value of the firm's production at equilibrium price. This echoes a well-known result that, in complete markets, shareholders would agree on profit maximization or, equivalently, on aggregate dividend maximization. Notice however that the result is not immediate in our setting, in which production, prices, and the manager's compensation are jointly determined in equilibrium. A priori shareholders' aggregate dividend is not equal nor proportional to the value of production, the difference between these two quantities corresponding to the value of manager's compensation.

Second, we show that the manager's compensation is necessarily linear or, equivalently, the manager is given a fixed share of production. Third, we show that the manager does not trade in the financial market. In fact, the manager would misreport the chosen plan unless at equilibrium the marginal value of her compensation is proportional to her marginal utility of consumption. It follows that her consumption profile coincides with her compensation. Intuitively, if this were not the case, the manager could increase her utility by announcing a given plan, implement a different one, and exploit the induced mispricing by trading on her private information. Hence, at equilibrium, the manager has no incentive to trade.

From the previous results we obtain a characterization of a family of manager-shareholders equilibria, if they exist. These results allow to construct our equilibrium by separating the manager's maximization problem from that of shareholders. The equilibrium production plan maximizes the manager's utility on the set of production plans. At the same time, it is the equilibrium production plan if the firm was run only by the shareholders, according to profit maximization.

We define the representative shareholder at equilibrium as a fictitious shareholder who - if endowed with the equilibrium production plan - would have no incentive to trade at equilibrium prices. We can then state a necessary and sufficient condition for the existence of a manager-shareholders 
equilibrium. We show that such equilibrium exists if and only if the manager has the same characteristics as the representative shareholder at equilibrium.

The properties of the representative agent at equilibrium have extensively been studied in the literature on aggregation of preferences and beliefs in asset markets. To derive some properties of the equilibrium manager as a function of shareholders' characteristics, we assume that all shareholders have same CRRA instantaneous utility function. The representative shareholder at equilibrium is then an expected utility maximizer with the same CRRA instantaneous utility function, whose belief is a function of shareholders' beliefs and discount rates and whose discount rate is time dependent or stochastic. In particular, when all shareholders and the manager share the same belief but differ in their time preference, the manager needs to have a declining discount rate. This is because aggregate consumption in the distant future is mostly in the hands of more patient individuals. When the horizon becomes arbitrarily large, the manager needs to behave as the most patient shareholder. An implication is that a manager whose discount rate is an average of shareholders' discount rates would take decisions that are too focused on the short-term relative to shareholders' best interest. Moreover, when shareholders have the same constant discount rate but different beliefs, the manager cannot have the same discount rate as shareholders. Rather, she needs to put a higher weight on distant consumption than any of the shareholders. Put differently, a manager with the same discount rate as the shareholders would be short-termist.

We think our results have important implications for the study of agency problems. Our setting is most closely related to a common agency problem, in which an agent faces multiple principals with possibly conflicting interests (as started by Bernheim and Whinston (1986)). Our key distinctive feature is to embed this problem in a general equilibrium setting, in which the asset market is used to mediate and possibly align shareholders' conflicting interests. In this perspective, we highlight the importance of modeling explicitly shareholder heterogeneity and the equilibrium process leading to the definition of a representative shareholder. In our model, the representative shareholder is defined at equilibrium, and as mentioned before, it is the representative shareholder at a production equilibrium when 
the firm is run only by shareholders according to profit maximization. As we show, the insights one would get by taking the representative shareholder as given would be different, and possibly misleading, especially in settings where shareholder heterogeneity is important.

We qualify the view that agency conflicts are minimized when the manager is also a shareholder, which has motivated the rise in stock compensation. Stock compensation indeed appears as necessary in our setting. At the same time, we emphasize that the manager must have the same characteristics as the representative shareholder. Otherwise, whatever fraction of the firm's share she owns, she would take decisions not unanimously supported by all shareholders.

In this respect, we view our results mostly as normative, emphasizing the conditions for the existence of a manager-shareholders equilibrium in which the manager's actions are perfectly aligned with shareholders' interests. From a positive viewpoint, we do not take the existence of such an equilibrium as granted. It may be very difficult or impossible to find a manager with the same characteristics as the representative shareholder. Under this perspective, one may interpret our results as suggesting the impossibility of reaching an equilibrium in which the production decision is delegated to a manager while serving shareholders' interests. However, we view this paper only as a starting point in addressing the question of delegation. A natural next step is to investigate whether richer contracts (that may include trading restrictions for the manager) together with possibly non-linear compensations may help achieving a manager-shareholders equilibrium. In a companion paper (Bianchi, Dana and Jouini (2020)), we define conditions for existence of a manager-shareholders equilibrium in a setting where trading restrictions can be imposed on the manager. We get back to this issue in more details in the concluding remarks.

Our paper builds on the literature on aggregation of preferences and beliefs in asset markets. ${ }^{1}$ Our focus on agency problems between a manager and shareholders is however novel in this literature. Similarly, we relate to the literature on asset pricing with production and agency costs. ${ }^{2}$ This

\footnotetext{
${ }^{1}$ Recent contributions include Detemple and Murthy (1994); Gollier and Zeckhauser (2005); Jouini and Napp (2007); Jouini, Marin and Napp (2010); Cvitanić, Jouini, Malamud and Napp (2012); Xiong and Yan (2010); Bhamra and Uppal (2014).

${ }^{2}$ See for example Dow, Gorton and Krishnamurthy (2005), Albuquerue and Wang
} 
literature typically abstracts from shareholder heterogeneity. Our analysis is complementary, as we abstract from asset pricing implications and focus on how to choose and remunerate a manager in a way to minimize agency costs.

Managerial compensation has generally been studied under the perspective of a representative shareholder (see e.g. Murphy (1999) and Murphy (2012) for reviews). We provide new insights by embedding the choice of the compensation in a stock market equilibrium with heterogeneous shareholders. In this spirit, Bolton, Scheinkman and Xiong (2006) consider a market with heterogeneous beliefs and short-selling constraints and show that shareholders may prefer short-term speculative strategies. ${ }^{3}$ In our model, the market is efficient and there is no resale, but (inefficient) shorttermism may arise as the result of shareholder heterogeneity.

We also relate to the literature on firms' objectives when shareholders are heterogeneous. Magill and Quinzii (2002) review fundamental problems posed by market incompleteness, as well as classic contributions addressing these problems. Bisin, Gottardi and Ruta (2016) study competitive equilibria in a production economy with incomplete markets and agency frictions and derive fundamental welfare properties. ${ }^{4}$ We instead focus on the design of the compensation scheme and keep shareholders' objective as simple as possible by assuming complete markets, or - more precisely - full spanning. As explained in Magill and Quinzii (2009), this assumption is typically much weaker than market completeness, and it means that it is possible to find a portfolio of assets that pays one unit if a given outcome for the firm is realized, and nothing otherwise.

The way information asymmetry is introduced makes our paper in line with the probability approach to general equilibrium developed by Magill and Quinzii (2009). Indeed, we assume that shareholders do not observe states of nature but only the production outcomes, so from their point of view, production plans only differ by the outcomes probability distribution. (2008), Gorton, He and Huang (2014).

${ }^{3}$ Alternative equilibrium models have instead focused on the labor market equilibrium (e.g. Gabaix and Landier (2008)) or on financial market equilibrium with a representative agent (e.g. Diamond and Verrecchia (1982)).

${ }^{4}$ Other recent contributions include Zame (2007), Demichelis and Ritzberger (2011), Magill, Quinzii and Rochet (2015), Crès and Tvede (2021). 
Accordingly, we consider contracts that are contingent on the possible realizations firm's production as opposed to being contingent on exogenous states of nature. ${ }^{5}$

\section{Model}

We consider a firm owned by a group of shareholders, who are heterogeneous in beliefs and time preferences, and run by a manager. The firm produces a non-storable consumption good, which we use as numeraire, according to a production plan $y$. This plan is a random process in which $y_{t}(\omega)$ defines the production of the firm at date $t$ in state $\omega$. There is a finite set of production and consumption dates $\mathbb{T}=\{0, \ldots, T\}$ or $\mathbb{T}=\{T\}$ when there is only one consumption/production date. ${ }^{6}$ The information structure is modeled by a filtered probability space $\left(\Omega,\left(\mathcal{F}_{t}\right)_{t \in \mathbb{T}}, P\right)$.

We denote by $X$ the space of production and consumption processes $x=\left(x_{0}, \ldots, x_{T}\right)$. For each $t, x_{t}$ is assumed to be $\mathcal{F}_{t}$ measurable so that $x_{t}$ only involves information up to date $t$. We denote by $X^{\prime}$ the space of state-price densities $p$ where, for a given date $t \in\{0, \ldots, T\}$ and a given state of the world $\omega \in \Omega, p_{t}(\omega)$ corresponds to the price as of $t=0$, of one unit of consumption at date $t$ in state $\omega$. For a given price process $p$, the value of the consumption process $x$ is $p \cdot x=\sum_{t=0}^{T} E\left[p_{t} x_{t}\right]$, where $E$ is the expectation operator under the probability $P$.

For these expectations to be well defined, we have to further impose that production and consumption processes in $X$ are such that $E\left|x_{t}\right|^{r}<\infty$ for all $t \in T$ and that price processes in $X^{\prime}$ are such that $E\left|p_{t}\right|^{r^{\prime}}<\infty$, where $r$ and $r^{\prime}$ are such that $1<r<\infty$ and $\frac{1}{r}+\frac{1}{r^{\prime}}=1 .^{7}$ We denote by $X_{+}$

\footnotetext{
${ }^{5}$ As underlined by Magill and Quinzii (2009) "That this assumption is realistic seems to be confirmed by the striking fact that the contracts which are used to finance investment and share production risks - bonds, equity and derivative securities - are either non contingent or based on realized profits and prices, rather than on exogenous events with fixed probabilities."

${ }^{6}$ For ease of presentation, all definitions and results are stated for $\mathbb{T}=\{0, \ldots, T\}$. The adaptation to the $\mathbb{T}=\{T\}$ is straightforward.

${ }^{7}$ The space $X$ equipped with the norm $\|x\|=\left(\sum_{t=0}^{T} E\left|x_{t}\right|^{r}\right)^{1 / r}$ is then a Banach space whose dual (the space of continuous linear forms on $X$ ) is $X^{\prime}$. In fact $X$ is $L^{r}$ space. It can be seen as $L^{r}(\hat{\Omega}, \widehat{\mathcal{F}}, P)$ where $(\widehat{\Omega}, \widehat{\mathcal{F}}, \widehat{P})$ is the direct sum of the probability spaces $\left(\Omega, \mathcal{F}_{t}, P\right)$, i.e. $\widehat{\Omega}$ is the disjoint union of $T+1$ many $\operatorname{copies}\left(\Omega_{t}\right)$ of $\Omega, \widehat{\mathcal{F}}$ is the $\sigma$-algebra
} 
and $X_{+}^{\prime}$ the set of nonnegative processes, respectively, in $X$ and $X^{\prime}$. For $Y \subset X$, we denote by $Y_{+}$the set $Y \cap X_{+}$.

In terms of notations, while $x$ and $y$ will be used to denote random consumption and production processes taking their values in $\mathbb{R}^{T+1}$, z will be used to denote vectors in $\mathbb{R}^{T+1}$ and, henceforth, generic values taken by $x$ or $y$. As usual, $x \leq x^{\prime}\left(x \ll x^{\prime}\right)$ means $x_{t} \leq x_{t}^{\prime}\left(x_{t}<x_{t}^{\prime}\right)$ almost everywhere for all $t$, and $x<x^{\prime}$ means $x \leq x^{\prime}$ and $x \neq x^{\prime}$. Finally, we denote by $\mu$ and $\mu^{t}$ the Lebesgue measure, respectively, on $\mathbb{R}$ and $\mathbb{R}^{t}$.

\subsection{Production}

We focus on the unique consumption good that is produced by the firm. Therefore, the choice of a production plan corresponds to the choice of a technology that transforms these inputs into units of consumption good across time and states of the world.

We let $Y \subset X$ denote the set of production plans. Denote with $N_{Y}(y)$ the normal cone of $Y$ at $y$,

$$
N_{Y}(y)=\left\{p \in X^{\prime}: p \cdot\left(y^{\prime}-y\right) \leq 0, \forall y^{\prime} \in Y\right\},
$$

which corresponds to the set of linear forms that reach their maximum on $Y$ at $y$. We will say that $y \in Y$ is positively exposed if there exists $p \gg 0$ such that $p \in N_{Y}(y)$. Note that a positively exposed production plan $y$ is efficient in the sense that it is not dominated by other feasible production plans : $\nexists y^{\prime} \in Y, y^{\prime}>y$. We denote by $\mathrm{Eff}^{+}(Y)$ the set of positively exposed production plans.

We say that $Y$ is smooth if, for all $\bar{y} \in \operatorname{Eff}^{+}(Y)$, there exists $p \gg 0$ such that $N_{Y}(\bar{y})=\{\lambda p: \lambda \geq 0\}$. This condition states that at positively exposed plans, the tangent cone (i.e. the polar of the normal cone) is a half space and it ensures that $Y$ has no outward kink.

We will say that $Y$ is strictly convex from above if for $\left(y_{1}, y_{2}\right) \in Y^{2}$ and $t \in(0,1)$, there exists $y \in Y$ such that $t y_{1}+(1-t) y_{2}<y{ }^{8}$

of sets $A \subset \widehat{\Omega}$ such that $A \cap \Omega_{t} \in \mathcal{F}_{t}$ for all $t$ and $\widehat{P}$ induces on each $\left(\Omega_{t},\left.\widehat{\mathcal{F}}\right|_{\Omega_{t}}\right)$, the initial probability $P$.

${ }^{8}$ Let us recall that $Y$ is said strictly convex when for all $\left(y_{1}, y_{2}\right) \in Y^{2}$ and $t \in(0,1)$, $t y_{1}+(1-t) y_{2} \in \operatorname{int}(y)$. It is immediate that strict convexity implies strict convexity 
We make the following assumptions:

\section{Assumption (P)}

(P1) $Y=K-X_{+}$where $K \subset X_{+}$and such that $0 \ll \varsigma \leq K \leq \Xi$ for some s and $\Xi$ in $X_{+}$,

(P2) $Y$ is closed, strictly convex from above and smooth,

(P3) If $y \in \mathrm{Eff}^{+}(Y)$, the random variable $y$ has a density $h_{y}$ with $h_{y}>0$, $\mu^{T+1}$-a.e. on $\mathbb{R}_{+}^{T+1}$.

Assumption (P1) implies the classical free disposal assumption, $Y-$ $X_{+} \subset Y$. It also provides an upper bound on $Y_{+}$. Finally, assumption (P1) ensures that utility maximization on $Y$ leads to plans $y$ such that $\varsigma \leq y \leq \Xi$. Note that under the free disposal assumption, strict convexity from above implies the convexity of $Y$. Assumption (P1) and (P2) are standard in the general equilibrium literature in finite dimension. Assumption (P3) states that for every positively exposed production plan, all trajectories taking positive values are possible. ${ }^{9}$ This implies that by observing a given trajectory of realizations $\left(y_{t}(\omega)\right)_{\{0, \ldots, T\}}$ of a given production process in $\operatorname{Eff}^{+}(Y)$, shareholders are not able to exclude any plan $y \in Y$ from the set of possibly chosen plans. This assumption underlies the information asymmetry between the manager and the shareholders in our model.

In order to illustrate Assumptions (P2) and (P3), we consider the following example, in which production and consumption only take place at date $T$.

Example 1 (Controlled Ito processes) Let $\left(W_{t}\right)_{t \in[0, T]}$ be a Brownian motion on some probability space $(\Omega, \mathcal{G}, P)$ and $\left(\mathcal{G}_{t}\right)_{t \in[0, T]}$ be the (augmented) filtration generated by $\left(W_{t}\right)_{t \in[0, T]}$. For a $\left(\mathcal{G}_{t}\right)_{t \in[0, T]}$-progressively measurable positive real valued process $\theta$ such that $\int_{0}^{T} \theta_{s}^{2}<\infty$ a.e., we define

from above. However strict convexity is a much stronger condition and requires, in particular, a nonempty interior.

${ }^{9}$ In this assumption $\mathbb{R}_{+}^{T+1}$ might be replaced by some $[A, B]^{T+1}$ for $0<A<B$. In this case, all the considered functions that are defined on $\mathbb{R}_{+}^{T+1}$ are replaced by functions that are only defined on $[A, B]^{T+1}$. 
$\left(y_{t}^{\theta}\right)$ as the solution of $d y_{t}^{\theta}=\mu\left(\theta_{t}\right) y_{t}^{\theta} d t+\theta_{t} y_{t}^{\theta} d W_{t}$ and $y_{0}^{\theta}=1$ where $^{10}$ $\mu(z)=1-\frac{(z-2)^{2}}{2+|z-2|}$. We denote by $\Theta$ the set of such processes $\theta$ and by $\mathcal{Y}$ the set of terminal values $y_{T}^{\theta}$ for $\theta \in \Theta$. We take $\mathbb{T}=\{T\}, \mathcal{F}=\mathcal{G}_{T}$, $X=L^{2}(\Omega, \mathcal{F}, P)$. It is shown in an Online Appendix that $Y=\mathcal{Y}-X_{+}$ satisfies (PQ) and (P3).

\subsection{Shareholders}

The firm is owned by a group of $N$ shareholders, $i=1, \ldots, N$. We denote with $\nu^{i}$ shareholder $i$ 's initial endowment of shares, and we assume $\nu^{i}>0$ for all $i$. Shareholders have no other endowments, and they are heterogeneous in their time preference rates $\rho^{i}$ assumed to be constant and in their subjective probabilities $Q^{i}$. All subjective probabilities are assumed to be equivalent to $P$ and we denote by $M^{i}$ the density of $Q^{i}$ with respect to $P$, $M^{i}=\frac{d Q^{i}}{d P}$.

A key ingredient in our analysis is that shareholders do not observe the plan $y=\left(y_{t}\right)_{t \in \mathbb{T}}$ chosen by the manager nor the state of the world $\omega$. At date $t$ and in state $\omega$, their information is given by the history of realizations $\left(y_{s}(\omega)\right)_{s=0, \ldots, t}$. As already mentioned, by Assumption P3, the observation of a given trajectory does not allow them to infer the chosen plan nor the state of the world. It follows that shareholders can only trade assets whose payoffs are contingent on the history of the production process $y$. More formally, let $\mathcal{C}$ be the set of contingent contracts $C: X_{+} \rightarrow X_{+}$ whose payoffs at date $t \in \mathbb{T}$ and for a given $y$ are of the form $c_{t}(\omega)=$ $C_{t}\left(y_{0}(\omega), \ldots, y_{t}(\omega)\right)$, for some measurable functions $C_{t}: \mathbb{R}_{+}^{t+1} \rightarrow \mathbb{R}_{+}$. Given $y$, shareholders only trade consumption processes in $\mathcal{C}(y)=\{C(y), C \in \mathcal{C}\}$.

All shareholders have the same consumption space $X_{+}$and they are assumed to be expected utility maximizers. The expected utility of shareholder $i$ for a contingent consumption plan $c$ is defined as

$$
U^{i}(c)=\sum_{t=0}^{T} \exp \left(-\rho^{i} t\right) E\left[M_{t}^{i} u\left(c_{t}\right)\right],
$$

\footnotetext{
${ }^{10}$ In this example, the instantaneous level of risk (volatility) is given by $\theta$ and the instantaneous rate of return (drift) is given by $\mu(\theta)$. The rate of return increases with the level of risk until a given risk level $\theta^{*}=2$ and then decreases. And when there is no risk, $\theta=0$, there is no return.
} 
in which $u$ is a CRRA instantaneous utility function (the same for all shareholders). ${ }^{11}$ That is

$$
u(x)=\frac{1}{\gamma} x^{\gamma},
$$

for some $\gamma<1$. We further assume the following:

\section{Assumption (C)}

1. For all $i, M^{i}$ and $M^{i} \varsigma^{\gamma-1}$ belong to $X^{\prime}$,

2. For all $i, M^{i} \varsigma^{\gamma}$ and $M^{i} \Xi^{\gamma}$ belong to $L^{1}\left(\Omega,\left(\mathcal{F}_{t}\right)_{t \in \mathbb{T}}, P\right)$.

Assumption (C) assures that shareholders' marginal utility is well defined in all directions and that their utility is well defined on $K$.

\subsection{Manager}

The firm is run by a manager. As mentioned, we do not model explicitly why shareholders need to delegate this choice to a manager. A standard argument is that they lack the time or the skills needed to implement the plan, which may require continuous adjustments over time. ${ }^{12}$

We assume that the manager is an expected utility maximizer with instantaneous utility $u$, as defined in (2), she has a constant time preference rate $\rho_{m}$ and a subjective probability $Q_{m}$ equivalent to $P$ with density $M_{m}$. Her expected utility of a contingent plan $c$ is therefore defined by

$$
U_{m}(c)=\sum_{t=0}^{T} \exp \left(-\rho_{m} t\right) E\left[M_{m, t} u\left(c_{t}\right)\right] .
$$

The manager is given a contract described by a compensation scheme $\Phi$ : $X_{+} \rightarrow X_{+}$. As shareholders can only observe the realized production, the compensation at date $t$ can only depend on the history of realizations

\footnotetext{
${ }^{11}$ Assuming that shareholders have no other endowments than their shares of the firm and that instantaneous utility functions are CRRA with homogeneous risk aversion parameters, will appear as crucial for the proof of existence and uniqueness of a production equilibrium as seen in Appendix 2.

${ }^{12}$ For instance, in Example 1, the manager chooses her risk exposure $\theta$ upon observing the signals $\left(y_{t}^{\theta}\right)$. That requires having the skills to assess the impact of the chosen $\theta$ on the dynamics of the production process as well as the time to continuously monitor the signals and adjust the risk exposure accordingly.
} 
$y_{0}(\omega), \ldots, y_{t}(\omega)$. That is, $\Phi(y)$ must be of the form

$$
\Phi_{t}(y(\omega))=\phi_{t}\left(y_{0}(\omega), \ldots, y_{t}(\omega)\right)
$$

for some $\phi_{t}: \mathbb{R}_{+}^{t+1} \rightarrow \mathbb{R}_{+}$assumed to be continuous almost everywhere.

Shareholders delegate to the manager the choice of the production plan. In the framework of Example 1, this means that the manager chooses the degree $\theta_{t}$ of risk exposition at each date $t$. In this respect, the example has some similarities with Sannikov (2008), with two important differences. First, our model has as many principals as the number of shareholders. Second, manager's choices in our case are not determined by an arbitrage between effort and compensation but through a more complex market mechanism, as we detail below.

To explain how the manager makes her choices, let us introduce the concept of indirect utility of production plans for a given price process. Given a price process $q$ and production plan $y$, let $V_{m}(y, q)$ be the maximal utility of the consumption processes that the manager can obtain by trading her compensation under her market constraint $c \in \mathcal{C}(y)$ and her budget constraint $q \cdot c \leq q \cdot \Phi(y)$,

$$
V_{m}(y, q)=\max \left\{U_{m}(c), c \in \mathcal{C}(y), q \cdot c \leq q \cdot \Phi(y)\right\}
$$

Given her compensation and a price process $q$, the manager chooses a production plan $y$ that maximizes her indirect utility $V_{m}(\cdot, q)$ over $Y$ and an optimal consumption plan $C_{m}(y)$. The manager announces the chosen plan $y$ to shareholders. who maximize the utility of their consumption plans under their market and budget constraints. From Assumption (P3), when $y$ is in $\operatorname{Eff}^{+}(Y)$ (which is the case for example if $\Phi$ is increasing), shareholders cannot verify the truth of the announcement and the manager can implement a plan with higher utility of consumption. Therefore the manager implements $y$ only if $U_{m}\left(C_{m}(y)\right)=\max _{y^{\prime} \in Y} U_{m}\left(C_{m}\left(y^{\prime}\right)\right)$.

For further use in the definition of equilibrium, let $V^{i}(y, q)$ be the maximal utility of the consumption processes that shareholder $i$ can obtain by trading her share of production under her market and budget constraints:

$$
V^{i}(y, q)=\max \left\{U^{i}(c), c \in \mathcal{C}(y), q \cdot c \leq \nu^{i}(q \cdot(y-\Phi(y)))\right\}
$$


where $\nu^{i}$ denotes her initial share and $y-\Phi(y)$ is the production left to shareholders after having paid the manager. Equation (4) and the corresponding shareholders' equation show how the manager and the shareholders assess the utility associated to the various alternative production plans. They compare their indirect utility under $y$ to the one they would have obtained under any alternative $y^{\prime} \in Y$, by taking prices $q$ as given. ${ }^{13}$

\subsection{Equilibrium}

Let us now define our concept of equilibrium between shareholders and the manager. We have in mind a setting with a large number of nonstrategic shareholders. We take a general equilibrium approach in which resource allocation is decentralized through prices and which we adapt so as to account for the information asymmetry between the manager and the shareholders.

Definition 1 A manager-shareholders equilibrium is defined by a compensation scheme $\Phi$, a production process $\hat{y} \in Y$, a list of contingent contracts $\left(\hat{C}^{i}\right)_{i}$, a contingent contract $\hat{C}_{m}$, and a price process $\hat{q}$ such that:

1. $\hat{c}^{i}=\hat{C}^{i}(\hat{y})$ maximizes $U^{i}(c)$ on $\left\{c \in \mathcal{C}(\hat{y}), \hat{q} \cdot c \leq \nu^{i}(\hat{q} \cdot(\hat{y}-\Phi(\hat{y})))\right\}$,

2. $\hat{c}_{m}=\hat{C}_{m}(\hat{y})$ maximizes $U_{m}(c)$ on $\{c \in \mathcal{C}(\hat{y}), \hat{q} \cdot c \leq \hat{q} \cdot \Phi(\hat{y})\}$,

3. $\sum_{i} \hat{c}^{i}+\hat{c}_{m}=\hat{y}$,

4. $V_{m}(\hat{y}, \hat{q})>V_{m}(y, \hat{q})$ for all $y \in Y \backslash\{\hat{y}\}$,

5. $U_{m}\left(\hat{C}_{m}(\hat{y})\right)=\max _{y \in Y} U_{m}\left(\hat{C}_{m}(y)\right)$,

6. $V^{i}(\hat{y}, \hat{q})>V^{i}(y, \hat{q})$ for all $y \in Y \backslash\{\hat{y}\}$.

For a given $\hat{y}$, conditions 1 to 3 define an exchange equilibrium (with market constraints given by $c \in \mathcal{C}(\hat{y}))$ between the manager and the shareholders with endowments $\Phi(\hat{y})$ and $\left(\nu^{i}(\hat{y}-\Phi(\hat{y}))_{i}\right.$. Together with point 4 ,

\footnotetext{
${ }^{13}$ Price taking is important to be able to define a consensus plan (see e.g. Grossman and Stiglitz (1980) for a discussion on price taking behaviors and unanimity). Price taking could also be derived by considering a setting with a large number of identical firms. The analysis would not be affected.
} 
they define a concept of equilibrium in the spirit of a production equilibrium (with market constraints) in which the choice of the plan is determined by the manager. Point 5 highlights a key distinctive feature of our equilibrium, relative to more standard agency problems in partial equilibrium. In our setting, the manager is not only choosing a production plan but also her consumption plan $C_{m}(y)$, possibly by trading in the asset market. This opens the possibility for the manager to announce a given plan, implement a different one, and increase her utility by trading on her private information and changing her consumption decisions. Our condition 5 implies that at equilibrium the manager has no incentive to misreport and so there is no asymmetric information between the manager and the shareholders. Alternatively, we may assume that the manager does not announce a plan and that the shareholders have expectations about manager's choice. Under this description of the model, condition 5 implies that shareholders' expectations are consistent with the plan actually chosen by the manager.

Point 6 means that, under $\hat{q}$, shareholders unanimously prefer $\hat{y}$ to any other plan $y$ in $Y$. It is known that when markets are complete and shareholders can directly choose production plans, unanimity holds. Our setting is different, since the decision is delegated to a manager and shareholders trade contingent claims on production. Note that the unanimity requirement does not seem too restrictive. It can be shown that under an additional technical condition (namely, the differentiability of $\Phi$ ), unanimity holds in our setting whenever there exists at least one shareholder who prefers $\hat{y}$ to any other plan $y$ in $Y$.

Given our equilibrium concept, we can highlight how the maximization problem of our manager expressed in Equation (4) differs from what appears in standard agency problems. First, our manager is maximizing her indirect utility, which depends not only on her action but also on the endogenous equilibrium price. Second, the manager's consumption may depend not only on her compensation but also on the possibility to trade in the asset market. This implies that in the equilibrium definition, we need to ensure that the manager has no incentive to announce a given plan, implement a different one, and increase her utility by trading on her private information and changing her consumption decisions. 


\section{$3 \quad$ Equilibrium Properties}

We start by analyzing the properties that equilibria should have.

\subsection{Profit is maximized}

We first show that at equilibrium, the price process $\hat{q}$ should be strictly positive, a standard property in equilibrium models. We then show that $\hat{y}$ should maximize $\hat{q} \cdot \hat{y}$ at price $\hat{q}$ on $Y$, a property that implies the efficiency of $\hat{y}$.

Proposition 1 Suppose Assumption (P) holds and let $\left(\Phi,\left(\hat{c}^{i}\right)_{i}, \hat{c}_{m}, \hat{q}, \hat{y}\right)$ be an equilibrium. Then $\hat{q} \gg 0$ and $\hat{q} \cdot \hat{y}=\max _{Y} \hat{q} \cdot y$. Hence, $\hat{y} \in \operatorname{Eff}^{+}(Y)$ and $N_{Y}(\hat{y})=\{\lambda \hat{q}, \lambda \geq 0\}$.

When $\hat{y}$ is implemented and under the price system $\hat{q}, \hat{q} \cdot \hat{y}$ corresponds to the market value of the firm's production. As already underlined, this resembles the well-known result that, in complete markets, shareholders would agree on profit maximization. At the same time, the result is not immediate in our setting. Profit maximization when the firm is run only by shareholders need not be equivalent to maximizing shareholders' aggregate dividend in our setting, as one needs to account also for the value of manager's compensation.

\subsection{The compensation is linear}

In this section we show that the equilibrium compensation is linear in the following sense.

Definition 2 We say that the compensation is linear if there exists $\alpha \in$ $[0,1]$ such that

$$
\Phi(y)=\alpha y \text { for all } y \in X_{+} \text {. }
$$

Such a compensation will be denoted $\Phi^{\alpha}$.

For this purpose, we have to introduce the following definition and additional assumption. For a given $\bar{y} \in Y$ and a given $t$, we define $Y(\bar{y}, t)=$ 
$\left\{y \in Y:\left(y_{0}, \ldots, y_{t}\right)=\left(\bar{y}_{0}, \ldots, \bar{y}_{t}\right)\right\}$, the set of production plans whose first $t+1$ coordinates coincide with those of $\bar{y}$.

Assumption (P4) For all $\bar{y} \in \operatorname{Eff}^{+}(Y)$ and $p \gg 0$ such that $N_{Y}(\bar{y})=$ $\{\lambda p: \lambda \geq 0\}, N_{Y(\bar{y}, t)}(\bar{y})=\left\{q \in X^{\prime}: \exists \lambda \geq 0, q_{s}=\lambda p_{s}\right.$ for $\left.s>t\right\}$, for all $t$.

This condition is in the spirit of the constraint qualification condition in convex optimization. ${ }^{14}$ This condition is obviously satisfied in Example 1 in which consumption only occurs at the terminal date.

The next theorem shows that at equilibrium, if it exists, the manager receives a linear compensation.

Theorem 2 Suppose assumptions (P1) to (P4) hold and let $\left(\Phi,\left(\hat{c}^{i}\right)_{i}, \hat{c}_{m}, \hat{q}, \hat{y}\right)$ be an equilibrium. Then there exists $0<\alpha<1$ such that $\Phi=\Phi^{\alpha}$.

To have an intuition of why the compensation scheme is linear, let us consider the case where $\phi_{t}$ is differentiable (actually, we show in the proof that this is the case almost everywhere). Notice that from our equilibrium definition, the value of the manager's compensation is maximal on $Y_{+}$at $\hat{y}$. Hence, the marginal value of the manager's compensation $\hat{q} \Phi^{\prime}(y)$ is proportional to the equilibrium price $\hat{q}$, which, by backward induction, gives that the compensation $\Phi(y)$ is linear. This implies that the incomes of the manager and of the shareholders are proportional to each other.

When prices are given the only feature of a compensation that matters is its market value. However, in our general equilibrium setting, prices are endogenous and depend in particular on the production plan chosen by the manager, which in turn depends on her compensation. Hence, the shape of the compensation matters and Theorem 2 states that this shape is necessarily linear.

We also notice that the equilibrium compensation is determined up to the scale parameter $\alpha$, that could be made arbitrarily close to 0 . In order to pin down $\alpha$, one could easily introduce a reservation utility for the manager $U^{*}$ and assume that shareholders minimize the compensation cost. It would then be immediate to show that the equilibrium level $\hat{\alpha}$ is fully determined by the condition $V^{m}(\hat{y}, \hat{q})=U^{*}$.

\footnotetext{
${ }^{14}$ It is easy to show that the inclusion $\left\{q \in X^{\prime}: \exists \lambda \geq 0, q_{s}=\lambda p_{s}\right.$ for $\left.s>t\right\} \subset$ $N_{Y(\bar{y}, t)}(\bar{y})$ is always satisfied.
} 


\subsection{The manager does not trade}

The manager does not trade in the financial market when her optimal demand coincides with her compensation. In this case, her consumption does not depend on asset prices, and therefore she does not benefit from announcing a given plan and implementing a different one. Our next result is that the manager truthfully reports the chosen plan $\hat{y}$ only if she has no incentive to trade her compensation $\Phi(\hat{y})$ in the financial market. Hence, at an equilibrium, if it exists, the manager's consumption $\hat{c}_{m}$ coincides with her compensation. We call this the no-trade result, which we state in the following:

Theorem 3 Suppose assumptions (P1) to (P4) hold. Let $\left(\Phi^{\alpha},\left(\hat{c}^{i}\right)_{i}, \hat{c}_{m}, \hat{q}, \hat{y}\right)$ be an equilibrium. Then the manager does not trade, in other words $\hat{c}_{m}=$ $\Phi^{\alpha}(\hat{y})$.

Let us give a sketch of the proof when there is only one period. Let $\left(\Phi^{\alpha},\left(\hat{c}^{i}\right)_{i}, \hat{c}_{m}, \hat{q}, \hat{y}\right)$ be an equilibrium. Then the manager receives $\alpha \hat{y}$. From assertion 2 in Definition 1, $\hat{c}_{m}$ maximizes $U_{m}(c)$ s.t. $c \in \mathcal{C}(\hat{y}), \hat{q} \cdot c \leq \alpha \hat{q} \cdot \hat{y}$. As we show that the constraint $c \in C(\hat{y})$ is not binding, the derivative of the utility of the manager at $\hat{c}_{m}$ is proportional to $\hat{q}$. Furthermore, from assertion 5 of Definition 1, $\hat{y}$ solves

$$
\max _{y \in Y} U_{m}\left(\hat{C}_{m}(y)\right)
$$

Condition (6) comes from the fact that by implementing a given $y$ the manager can change her consumption profile $\hat{C}_{m}(y)$ and that at equilibrium she does not misreport her choice. From the first order conditions, the derivative of $U_{m}\left(\hat{C}_{m}(y)\right)$ at $\hat{y}$ is also proportional to $\hat{q}$. Hence the two derivatives at $\hat{y}$ must be proportional. As the marginal utility appears on both sides, we have that $\hat{C}_{m}$ has a constant derivative. Therefore for some $a>0, \hat{C}_{m}(y)=a y$ for all $y$. From the manager's budget constraint $a \hat{q} \cdot \hat{y}=\alpha \hat{q} \cdot \hat{y}$, hence $a=\alpha$ which means that the manager does not trade.

Notice that neither the no trade nor the linear compensation are requirements of the model, they result from the agency problem in our general equilibrium setting. 


\subsection{Relation to the production equilibrium}

The aim of this subsection is to characterize the set of manager-shareholders equilibria. To this end, we use the no-trade result to separate, for a given share $\alpha$ of production given to the manager, the equilibrium construction into two parts. On one side, the manager chooses the production plan $\hat{y}$ that maximizes $U_{m}(\alpha y)$ on $Y$. On the other, the shareholders choose a production plan as if they were running by themselves according to profit maximization, a firm with production set $(1-\alpha) Y$. At equilibrium, the manager's and shareholders' choices should coincide. In order to state our result, let us first recall the concept of production equilibrium associated to a given production set. Note that in its definition, shareholders are not constrained anymore to trade contingent claims that depend on production plans, they may trade any contingent claim.

Definition 3 A production equilibrium associated to the production set $\mathcal{Y}$ is given by a production process $y^{*}, y^{*} \in \mathcal{Y}$, a set of individual consumption processes $\left(c^{* i}\right)_{i} \in X^{N}$ and a price process $q^{*} \in X^{\prime}$ such that

1. $c^{* i}=\operatorname{argmax} U^{i}(c), q^{*} \cdot c \leq \nu^{i}\left(q^{*} \cdot y^{*}\right)$ for all $i$,

2. $y^{*}=\arg \max y q^{*} \cdot y$,

3. $\sum c^{* i}=y^{*}$.

The following theorem shows the existence and uniqueness of such a production equilibrium.

Theorem 4 Suppose assumptions (P1), (P2) and (C) hold.

1. There exists a unique production equilibrium associated to $Y$ denoted by $\left(\left(c^{* i}\right)_{i}, q^{*}, y^{*}\right)$, with $q^{*} \gg 0$.

2. For any $\left.\alpha \in(0,1),\left((1-\alpha) c^{* i}\right)_{i}, q^{*},(1-\alpha) y^{*}\right)$ is the unique production equilibrium associated to $(1-\alpha) Y$.

The second assertion is a direct consequence of the first one using the homogeneity of utilities. In both assertions, equilibrium prices are defined 
up to a multiplicative constant. The proof of Theorem 4 may be found in Appendix 2.

In order to establish the link between the production equilibrium and our concept of manager-shareholders equilibrium, we need the following additional assumption.

Assumption (P5) The filtration $\mathcal{F}$ is generated by any $y \in \operatorname{Eff}^{+}(Y)$.

This is a completeness assumption which states that the market generated by all the contracts that are contingent on the values taken by $y$ is complete when $y \in \mathrm{Eff}^{+}(Y)$ and, in particular, for $y=y^{*}$. Indeed, as the production equilibrium plan $y^{*}$ is profit maximizing and the equilibrium price $q^{*}$ is strictly positive, $y^{*} \in \mathrm{Eff}^{+}(Y)$. Under Assumption (P5), the first item of the definition of a production equilibrium is equivalent to

$$
c^{* i}=\operatorname{argmax} U^{i}(c), c \in \mathcal{C}\left(y^{*}\right), q^{*} \cdot c \leq \nu^{i}\left(q^{*} \cdot y^{*}\right), \text { for all } i,
$$

which means that under Assumption (P5), the standard concept of production equilibrium is equivalent to a concept of production equilibrium with market constraints. ${ }^{15}$

From Theorems 2, 3 and Proposition 1, we obtain a characterization of a manager-shareholders equilibrium.

Theorem 5 Suppose assumptions (P1) to (P5) and (C) hold. Let $\alpha \in$ $(0,1)$ be given. The list $\left(\Phi^{\alpha},\left(\hat{c}^{i}\right)_{i}, \alpha \hat{y}, \hat{q}, \hat{y}\right)$ is a manager-shareholders equilibrium if and only if

1. the production plan $\hat{y}$ maximizes $U_{m}(y)$ over $Y$,

2. the triple $\left(\left(\hat{c}^{i}\right)_{i}, \hat{q},(1-\alpha) \hat{y}\right)$ is the production equilibrium associated to $(1-\alpha) Y$. Therefore, $\hat{c}^{i}=(1-\alpha) c^{* i}$ for all $i, \hat{q}=\nu q^{*}$ for some $\nu>0$ and $\hat{y}=y^{*}$.

\footnotetext{
${ }^{15}$ Note that this assumption is not necessary when all agents have homogeneous beliefs. Indeed, in such a setting, it is easy to show that even if the agents can trade all possible contracts and not only those that are contingent on the values taken by $y$, they will only trade such contingent contracts (see Appendix 2).
} 


\section{Equilibrium Manager}

We next show that, for any $0<\alpha<1$, at the production equilibrium associated to $(1-\alpha) Y$, there is a representative shareholder independent of $\alpha$ and we fully describe her characteristics. As standard, this is a fictitious shareholder who - if endowed with the entire production - would have no incentive to trade at equilibrium prices. We then derive the main result of the paper: a necessary and sufficient condition for the existence of managershareholders equilibria is that the manager has the same characteristics as the representative shareholder at equilibrium. We refer to such manager as an equilibrium manager.

We start by characterizing the representative shareholder in our setting. Let

$$
\Lambda=\left\{\lambda \in \mathbb{R}_{+}^{n}: \sum_{i}\left(\lambda^{i}\right)^{\frac{1}{1-\gamma}}=1\right\},
$$

and $N(\lambda)$ be defined by

$$
N_{t}(\lambda)=\left(\sum_{i}\left(\lambda^{i} M_{t}^{i} \exp \left(-\rho^{i} t\right)\right)^{\frac{1}{1-\gamma}}\right)^{1-\gamma} .
$$

Theorem 6 Suppose assumptions (P1), (P2) and $(C)$ hold. Let $\left(\left(c^{* i}\right)_{i}, q^{*}, y^{*}\right)$ be the production equilibrium associated to $Y$. Then:

1. There exists a unique vector of equilibrium utility weights $\left(\lambda^{* i}\right)$ in $\Lambda$ and $\nu>0$ such that

$$
\lambda^{* i} M_{t}^{i} \exp \left(-\rho^{i} t\right) u^{\prime}\left(c_{t}^{* i}\right)=\nu q_{t}^{*} \text { for all } t \text { and all } i \text {. }
$$

2. There is a representative shareholder with instantaneous utility $u$, a density $\tilde{M}$ and a nonnegative discount rate process $\tilde{\rho}$ uniquely determined by:

$$
\exp \left(-\tilde{\rho}_{t} t\right) \tilde{M}_{t}=N_{t}\left(\lambda^{*}\right)
$$

3. The belief and discount rate of the representative shareholder at the production equilibrium associated to $(1-\alpha) Y$ are independent of $\alpha$.

Using Theorem 5 and Theorem 6 , we can now state the main result of 
the paper. The following theorem shows that a manager-shareholders equilibrium exists if and only if the manager has the same belief and discount rate as the representative shareholder.

Theorem 7 Suppose assumptions (P1) to (P5) and $(C)$ hold. Let $\left(\left(c^{* i}\right)_{i}, q^{*}, y^{*}\right)$ be the production equilibrium associated to $Y$. The family parametrized by $\left.0<\alpha<1,\left(\Phi^{\alpha},\left((1-\alpha) c^{* i}\right)\right)_{i}, \alpha y^{*}, q^{*}, y^{*}\right)$ is the unique family of managershareholder equilibria if and only if $\left(\rho_{m}, M_{m}\right)$ satisfies

$$
\exp \left(-\rho_{m} t\right) M_{m, t}=\exp \left(-\tilde{\rho}_{t} t\right) \tilde{M}_{t} \text { for all } t \in \mathbb{T}
$$

which gives $M_{m, t}=\tilde{M}_{t}$ and $\rho_{m}=\tilde{\rho}_{t}$ for all $t \in \mathbb{T}$.

\section{Implications}

In this section we discuss some implications of Theorem 7 in terms of the characteristics (belief and discount rate) of the equilibrium manager as a function of shareholders' characteristics.

\subsection{Homogeneous shareholders}

If all the shareholders have the same belief $M$ and the same discount rate $\rho$ then, $N_{t}(\lambda)=M_{t} \exp (-\rho t)$, which does not depend on $\lambda$. By condition 8, we have $\tilde{\rho}=\rho$ and $\tilde{M}=M$ and, by condition 9 , the equilibrium exists if and only if the manager has the same belief and discount rate as the shareholders.

\subsection{Heterogeneous discount rates}

Suppose that all shareholders share the same belief as the manager, that is $M^{i}=M_{m}$ for all $i$, but they have different discount rates. Then by condition (8) we have $\tilde{M}=M_{m}$ and the discount rate of the manager is given by

$$
\exp \left(-\rho_{m} t\right)=\left(\sum_{i}\left(\lambda^{* i} \exp \left(-\rho^{i} t\right)\right)^{\frac{1}{1-\gamma}}\right)^{1-\gamma}
$$


This gives

$$
\rho_{m}=\frac{1}{t} \int_{0}^{t} \rho(s) d s,
$$

where $\rho(s)$ is the solution of

$$
\exp \left(-\int_{0}^{t} \rho(s) d s\right)=\left(\sum_{i}\left(\lambda^{* i} \exp \left(-\rho^{i} t\right)\right)^{\frac{1}{1-\gamma}}\right)^{1-\gamma}
$$

Using the same argument as in Nocetti, Jouini and Napp (2008), we obtain that $\rho(t)$ is decreasing with $\lim _{t \rightarrow \infty} \rho(t)=\inf \rho^{i}$. This means that there is no constant solution $\rho_{m}$ to equation (10) and that the manager has necessarily a decreasing discount rate. This is due to the fact that aggregate consumption in the distant future is mostly in the hands of more patient shareholders. This is shown in the following proposition.

Proposition 8 When all shareholders (including the manager) have the same belief $M$ and $\rho^{i} \neq \rho^{j}$ for some $(i, j)$, the equilibrium manager's discount rate must be decreasing over time.

A similar insight is derived by Gollier and Zeckhauser (2005). They focus on Pareto efficient allocations in a setting with no uncertainty and no production, and show that the representative shareholder has a decreasing discount rate. ${ }^{16}$ Similarly, in a continuous time equilibrium setting with heterogeneous beliefs and discount rates, Jouini et al. (2010) show that the representative shareholder discount rate cannot be constant and converges asymptotically to the lowest individual discount rate. In our setting, this means that the equilibrium manager behaves in the long run as the most patient shareholder. ${ }^{17}$

Note that we assumed in Section 2.3 that the manager has a constant discount rate. If we maintain this assumption, Proposition 8 can be seen as an impossibility result: in a setting where all the shareholders have the same belief and where at least two shareholders have different discount

\footnotetext{
${ }^{16}$ They show this is the case when all agents have constant discount rates and decreasing absolute risk aversions, which is the case in our framework.

${ }^{17}$ We cannot derive such a result directly from the equations above because our model has a finite horizon and changing $T$ leads to a possible change in the equilibrium weights $\left(\lambda^{* i}\right)$. In Appendix 3, we show that $\rho(T)$ approaches inf $\rho^{i}$ when $T$ is sufficiently large in a sense made precise.
} 
rates, whatever the belief and the constant discount rate of the manager, there is no manager-shareholders equilibrium.

\subsection{Heterogeneous beliefs}

Suppose instead that all shareholders have the same constant discount rate but different beliefs and $\gamma \neq 0$. Then by condition (9) the belief and discount rate of the manager should satisfy

$$
\exp (-\rho t)\left(\sum_{i}\left(\lambda^{* i} M_{t}^{i}\right)^{\frac{1}{1-\gamma}}\right)^{1-\gamma}=\exp \left(-\rho_{m} t\right) M_{m, t} .
$$

For $\gamma \leq 0$, we have (see Appendix 1)

$$
E_{t}\left[\left(\sum_{i}\left(\lambda^{* i} M_{t+1}^{i}\right)^{\frac{1}{1-\gamma}}\right)^{1-\gamma}\right] \leq\left(\sum_{i}\left(\lambda^{* i} M_{t}^{i}\right)^{\frac{1}{1-\gamma}}\right)^{1-\gamma} .
$$

When applied to equation (11), this leads to $\rho_{m} \geq \rho$, the inequality being strict when there are at least two shareholders with different beliefs and $\gamma \neq 0$. The equilibrium manager has a higher discount rate than any of the shareholder when $\gamma \leq 0$. The opposite inequality, $\rho_{m} \leq \rho$ is obtained for $\gamma>0$. We can state the following proposition.

Proposition 9 If $\gamma \neq 0$, when all shareholders have the same discount rate $\rho$ and $M^{i} \neq M^{j}$ for some $(i, j)$, then we must have $\rho_{m}>\rho$ when $\gamma<0$ and $\rho_{m}<\rho$ when $\gamma>0$.

If we introduce the consumption discount rate $\rho_{m, c}$, defined as

$$
\exp \left(-\rho_{m} t\right) u\left(c_{t}\right)=u\left(\exp \left(-\rho_{m, c} t\right) c_{t}\right)
$$

then $\rho_{m, c}=\frac{1}{\gamma} \rho_{m}$ and the manager's consumption discount rate is always lower than the shareholders common discount rate. That is, the manager puts more weight on distant consumption/production than any of the shareholders.

\section{Normal belief heterogeneity}


In order to derive some properties about the equilibrium manager belief, let us specify further shareholders' beliefs. Let us assume that $T=1$ and that the production set is characterized by a given random variable with normal distribution $\tilde{x} \sim \mathcal{N}\left(\mu, \sigma^{2}\right)$. For instance, this could correspond to the situation where the manager chooses the degree of exposure (level of volatility) to a given exogenous risk, the mean return being a function of the chosen degree of exposure.

Let us assume that all shareholders agree about the normality of $\tilde{x}$ as well as about its variance $\sigma^{2}$ but that shareholder $i$ believes that $\tilde{x} \sim$ $\mathcal{N}\left(\mu_{i}, \sigma^{2}\right)$, we have then

$$
M^{i}=\frac{\exp -\frac{\left(x-\mu_{i}\right)^{2}}{2 \sigma^{2}}}{\exp -\frac{(x-\mu)^{2}}{2 \sigma^{2}}}
$$

Let us assume that the distribution of the $\mu_{i}$ 's is symmetric with respect to the objective $\mu$ which is the case, for instance, with a large number of shareholders when the $\mu_{i}$ 's result from the observation of a noisy signal around $\mu$. Let us also assume that the $\lambda^{* i}$ as a function of $\mu_{i}$ is also symmetric with respect to $\mu .{ }^{18}$ We have

$$
\exp (-\rho)\left(\sum_{i}\left(\lambda^{* i} M^{i}\right)^{\frac{1}{1-\gamma}}\right)^{1-\gamma}=\exp \left(-\rho_{m}\right) M_{m}
$$

Since $\sum_{i}\left(\lambda^{* i} M_{t}^{i}\right)^{\frac{1}{1-\gamma}}$ is symmetric with respect to $\mu$ and increasing after $\mu$, it is easy to show that manager's belief is a mean preserving spread of the objective belief. Let $E^{m}$ and $\operatorname{Var}^{m}$ denote respectively the expected value and the variance from the manager point of view, $Q_{m}$ and $Q^{i}$ the manager's and shareholders' subjective probabilities and $\preceq_{S S D}$ second order stochastic dominance. We have

$$
E^{m}[\tilde{x}]=\mu, \operatorname{Var}^{m}[\tilde{x}]>\sigma^{2} \text { and } Q_{m} \preceq_{S S D} Q=\frac{1}{N} \sum_{i} Q^{i} .
$$

Therefore, the equilibrium manager overestimates the level of exogenous risk.

\footnotetext{
${ }^{18}$ The distribution of the $\left(\lambda^{* i}\right)$ depends on the distribution of shares $\left(\nu^{i}\right)$. This assumption is then an assumption on the distribution of shares.
} 


\subsection{Heterogeneous discount rates and beliefs}

We now consider a setting in which both beliefs and discount rates are heterogeneous. In this section we set $\gamma=0$ (logarithmic utility). From equation (9) we have

$$
\exp \left(-\rho_{m} t\right) M_{m, t}=\sum_{i} \lambda^{* i} \exp \left(-\rho^{i} t\right) M_{t}^{i} \text {, for every } t \in \mathbb{T},
$$

which leads to the following proposition.

Proposition 10 Suppose that $\gamma=0$ and $M_{s}^{i} \neq M_{s}^{j}$ for some $(i, j)$ and some $s \in T$. If the number of dates $t \in T$ such that $t>s$ is not smaller than the number of shareholders with distinct discount rates, then the equilibrium manager must have a non deterministic discount rate.

Similarly to Proposition 8, Proposition 10 can be seen as an impossibility result if one maintains the assumption that manager has a constant discount rate. In a logarithmic setting where at least two shareholders have different beliefs and with a sufficiently large number of dates, whatever the belief and the constant discount rate of the manager, there is no manager-shareholders equilibrium.

\section{Conclusion and Extensions}

A key result of our analysis is that a manager-shareholders equilibrium exists if and only if the manager has the same characteristics as the representative shareholder at equilibrium. This result implies a precise mapping between shareholders' and manager's characteristics, as we have characterized in Section 5. The flip side of these results is that finding an equilibrium manager may be difficult in settings in which such characteristics are hard to observe or in which they are constrained to be "similar" to those of shareholders.

In the context of Proposition 8, for example, one can see that it is not possible to find an equilibrium manager in the set of shareholders with constant discount rates, even though all shareholders have constant discount rates. Similarly, in Proposition 9, the equilibrium manager cannot 
be defined among shareholders with discount rate $\rho$, even though all shareholders have discount rate $\rho$. An implication is that, in order to overcome such impossibility, it is necessary to enrich the contracting space and allow shareholders to restrict the manager' ability to trade in the asset market. In such a framework and in a companion paper (Bianchi et al. (2020)), we investigate whether a manager-shareholders equilibrium could be achieved by imposing trading restrictions to the manager. We also analyze how the equilibrium compensation could be used to align the marginal utility of the manager to that of the representative shareholder at the equilibrium plan, and whether that would require departing from the linear compensation found in Section 3.

\section{References}

Albuquerue, R. and Wang, N. (2008), 'Agency conflicts, investment, and asset pricing', Journal of Finance 63(1), 1-40.

Berle, A. A. and Means, G. G. C. (1932), The modern corporation and private property, New York: Macmillan.

Bernheim, B. D. and Whinston, M. D. (1986), 'Common agency', Econometrica: Journal of the Econometric Society pp. 923-942.

Bhamra, H. S. and Uppal, R. (2014), 'Asset prices with heterogeneity in preferences and beliefs', Review of Financial Studies 27(2), 519-580.

Bianchi, M., Dana, R.-A. and Jouini, E. (2020), 'Equilibrium ceo contract with belief heterogeneity', Available at SSRN 3761935.

Bisin, A., Gottardi, P. and Ruta, G. (2016), 'Equilibrium corporate finance', Available at SSRN 2815066.

Bolton, P., Scheinkman, J. and Xiong, W. (2006), 'Executive compensation and short-termist behaviour in speculative markets', Review of Economic Studies 73(3), 577-610.

Crès, H. and Tvede, M. (2021), Democracy, the market, and the firm, Oxford University Press, forthcoming. 
Cvitanić, J., Jouini, E., Malamud, S. and Napp, C. (2012), 'Financial markets equilibrium with heterogeneous agents', Review of Finance 16(1), 285-321.

Dana, R.-A. (1995), 'An extension of milleron, mitjushin and polterovich's result', Journal of Mathematical Economics 24(3), 259-269.

Demichelis, S. and Ritzberger, K. (2011), 'A general equilibrium analysis of corporate control and the stock market', Economic theory 46(2), 221254 .

Detemple, J. and Murthy, S. (1994), 'Intertemporal asset pricing with heterogeneous beliefs', Journal of Economic Theory 62(2), 294-320.

Diamond, D. W. and Verrecchia, R. E. (1982), 'Optimal managerial contracts and equilibrium security prices', Journal of Finance pp. 275287.

Dow, J., Gorton, G. and Krishnamurthy, A. (2005), 'Equilibrium investment and asset prices under imperfect corporate control', American Economic Review 95(3), 659-681.

Gabaix, X. and Landier, A. (2008), 'Why has ceo pay increased so much?', Quarterly Journal of Economics 123(1), 49-100.

Gollier, C. and Zeckhauser, R. (2005), 'Aggregation of heterogeneous time preferences', Journal of Political Economy 113(4), 878-896.

Gorton, G. B., He, P. and Huang, L. (2014), 'Agency-based asset pricing', Journal of Economic Theory 149, 311-349.

Grossman, S. J. and Stiglitz, J. E. (1980), 'Stockholder unanimity in making production and financial decisions', Quarterly Journal of Economics 94(3), 543-566.

Jensen, M. C. and Meckling, W. H. (1976), 'Theory of the firm: Managerial behavior, agency costs and ownership structure', Journal of Financial Economics 3(4), 305-360. 
Jouini, E., Marin, J.-M. and Napp, C. (2010), 'Discounting and divergence of opinion', Journal of Economic Theory 145, 830-859.

Jouini, E. and Napp, C. (2007), 'Consensus consumer and intertemporal asset pricing with heterogeneous beliefs', Review of Economic Studies 74(4), 1149-1174.

Magill, M. and Quinzii, M. (2002), Theory of incomplete markets, Vol. 1, MIT press.

Magill, M. and Quinzii, M. (2009), 'The probability approach to general equilibrium with production', Economic Theory 39(1), 1-41.

Magill, M., Quinzii, M. and Rochet, J.-C. (2015), 'A theory of the stakeholder corporation', Econometrica 83(5), 1685-1725.

Murphy, K. (2012), 'Executive compensation: Where we are, and how we got there', Handbook of the Economics of Finance. Elsevier Science North Holland .

Murphy, K. J. (1999), 'Executive compensation', Handbook of labor economics $3,2485-2563$.

Nocetti, D., Jouini, E. and Napp, C. (2008), 'Properties of the social discount rate in a benthamite framework with heterogeneous degrees of impatience', Management Science 54(10), 1822-1826.

Sannikov, Y. (2008), 'A continuous-time version of the principal-agent problem', Review of Economic Studies 75(3), 957-984.

Xiong, W. and Yan, H. (2010), 'Heterogeneous expectations and bond markets', Review of Financial Studies 23(4), 1433-1466.

Zame, W. R. (2007), 'Incentives, contracts, and markets: A general equilibrium theory of firms 1', Econometrica 75(5), 1453-1500. 


\section{Appendices}

\subsection{Appendix 1. Proofs}

The following Lemma will be useful in the next. In particular, when some consumption plan $c$ does not satisfy the market constraints $c \in \mathcal{C}(y)$ for some $y \in Y$, the Lemma establishes that the market constraints are satisfied for some slight perturbation of $y$.

Lemma 11 For $\left(x, x^{\prime}\right) \in X_{+}^{2}$ and $\varepsilon>0$, there exists $x^{\prime \prime} \in X_{+}$such that $\left\|x^{\prime}-x^{\prime \prime}\right\|<\varepsilon$ and $\mathcal{C}(x) \subset \mathcal{C}\left(x^{\prime \prime}\right)$. Furthermore,

1. if $x$ and $x^{\prime}$ are in $Y$, we can take $x^{\prime \prime}$ such that $x^{\prime \prime} \in Y_{+}$,

2. if $x_{\tau}=x_{\tau}^{\prime}$ for $\tau=0, \ldots, t$ then we can take $x^{\prime \prime}$ such that $x_{\tau}=x_{\tau}^{\prime}=x_{\tau}^{\prime \prime}$ for $\tau=0, \ldots, t$.

Proof. Let $\left(x, x^{\prime}\right) \in X_{+}^{2}$ and let $\alpha>0$. Let $s_{n}=n+1$ for $n \geq 0$ and $s_{n}=\frac{1}{-n+1}$ for $n \leq 0$. The family $S=\left(s_{n}\right)_{n \in \mathbb{Z}}$ is an ordered family with $\lim _{\infty} s_{n}=\infty$ and $\lim _{-\infty} s_{n}=0$. Let $z$ and $z^{\prime}$ be nonnegative real numbers. If $z^{\prime}>0$, there exits $n \in \mathbb{Z}$ such that $s_{n} \leq z^{\prime}<s_{n+1}$ and we define $h$ by $h\left(z, z^{\prime}\right)=s_{n}-\left(s_{n}-s_{n-1}\right) \frac{z}{1+z}$. We have $0<s_{n-1}<h\left(z, z^{\prime}\right) \leq$ $s_{n} \leq z^{\prime}$ and $\left|h\left(z, z^{\prime}\right)-z^{\prime}\right|<2$. If $z^{\prime}=0$, we take $h(z, 0)=0$ and we still have $h\left(z, z^{\prime}\right) \leq z^{\prime}$. If $0<\epsilon<1$ is given and if $\epsilon z+(1-\epsilon) z^{\prime} \neq 0$, then $\left|h\left(z, \epsilon z+(1-\epsilon) z^{\prime}\right)-z^{\prime}\right|<2+\epsilon\left|z-z^{\prime}\right|$. If $\epsilon z+(1-\epsilon) z^{\prime}=0$ then $z=z^{\prime}=0$ and we still have $\left|h\left(z, \epsilon z+(1-\epsilon) z^{\prime}\right)-z^{\prime}\right|<2+\epsilon\left|z-z^{\prime}\right|$. Let us suppose now that we know $h\left(z, \epsilon z+(1-\epsilon) z^{\prime}\right)$ without knowing $z$ nor $z^{\prime}$. If $h\left(z, \epsilon z+(1-\epsilon) z^{\prime}\right)=0$ we necessarily have $\epsilon z+(1-\epsilon) z^{\prime}=0$ and from there $z=0$. If $h\left(z, \epsilon z+(1-\epsilon) z^{\prime}\right)>0$, the inequalities $s_{n-1}<$ $h\left(z, \epsilon z+(1-\epsilon) z^{\prime}\right) \leq s_{n}$ uniquely define a pair $\left(s_{n}, s_{n-1}\right)$ and from there $z$ is uniquely determined by the equation $h\left(z, z^{\prime}\right)=s_{n}-\left(s_{n}-s_{n-1}\right) \frac{z}{1+z}$. Now, let us define $x^{\prime \prime}$ by $x_{t}^{\prime \prime}=\epsilon h\left(\frac{x_{t}}{\epsilon}, \epsilon \frac{x_{t}}{\epsilon}+(1-\epsilon) \frac{x_{t}^{\prime}}{\epsilon}\right)$. We have $\left|x_{t}^{\prime \prime}-x_{t}^{\prime}\right|<$ $\epsilon\left(2+\epsilon\left|\frac{x_{t}}{\epsilon}-\frac{x_{t}^{\prime}}{\epsilon}\right|\right) \leq \epsilon\left(2+\left|x_{t}-x_{t}^{\prime}\right|\right)$ and if we take $\epsilon$ sufficiently small, we have $\left\|x^{\prime}-x^{\prime \prime}\right\|<\varepsilon$. Furthermore, knowing $x_{t}^{\prime \prime}$ permits to determine $x_{t}$ and we have $\mathcal{C}(x) \subset \mathcal{C}\left(x^{\prime \prime}\right)$. Finally, we have seen that $h\left(z, z^{\prime}\right) \leq z^{\prime}$ and then $0 \leq x^{\prime \prime} \leq \epsilon x+(1-\epsilon) x^{\prime} \in Y_{+}$by convexity of $Y_{+}$. We have then $x^{\prime \prime} \in Y_{+}$.

It is clear that when $x_{\tau}=x_{\tau}^{\prime}$, it suffices to take $x_{\tau}^{\prime \prime}=x_{\tau}=x_{\tau}^{\prime}$ instead of $x_{\tau}^{\prime \prime}=\epsilon h\left(\frac{x_{\tau}}{\epsilon}, \epsilon \frac{x_{\tau}}{\epsilon}+(1-\epsilon) \frac{x_{\tau}^{\prime}}{\epsilon}\right)$. 
Proof of Proposition 1. Let us first show that $\hat{q} \gg 0$. Indeed if for some $t, \hat{q}_{t}=0$ on a set $A$ of positive measure, the shareholders' and manager's demand could be arbitrarily large on $A$ violating assertion 3 of the definition of equilibrium.

Assume that there exists $y^{\prime} \in Y_{+}$such that $\hat{q} \cdot y^{\prime}>\hat{q} \cdot \hat{y}$. By Lemma 11, there exists $y^{\prime \prime}$ close to $y^{\prime}$ such that $\hat{q} \cdot y^{\prime \prime}>\hat{q} \cdot \hat{y}$ and $\mathcal{C}(\hat{y}) \subset \mathcal{C}\left(y^{\prime \prime}\right)$. We have then either $\hat{q} \cdot \Phi\left(y^{\prime \prime}\right)>\hat{q} \cdot \Phi(\hat{y})$ or $\hat{q} \cdot\left(y^{\prime \prime}-\Phi\left(y^{\prime \prime}\right)\right)>\hat{q} \cdot(\hat{y}-\Phi(\hat{y}))$. From the definition of the indirect utility, we have either $V_{m}\left(y^{\prime \prime}, \hat{q}\right)>V_{m}(\hat{y}, \hat{q})$ or $V^{i}\left(y^{\prime \prime}, \hat{q}\right)>V^{i}(\hat{y}, \hat{q})$ which violates assertion 4 or assertion 6 of the definition of equilibrium. Therefore, $\hat{q} \cdot \hat{y}=\max _{Y_{+}} \hat{q} \cdot \Phi(y)$. Since $\hat{q} \gg 0, \hat{q} \cdot \hat{y}=$ $\max _{Y} \hat{q} \cdot \Phi(y), \hat{y} \in \operatorname{Eff}^{+}(Y)$ and $N_{Y}(\hat{y})=\{\lambda \hat{q}, \lambda \geq 0\}$.

Proof of Theorem 2. The proof requires different steps.

SteP 1. Date $-T$ compensation $z_{T} \rightarrow \phi_{T}\left(z_{0}, \ldots, z_{T}\right)$ and net production $z_{T} \rightarrow z_{T}-\phi_{T}\left(z_{0}, \ldots, z_{T}\right)$ are nondecreasing in $z_{T}$.

The idea of this step is very simple. If the compensation is lower at $z^{\prime}$ than at $z<z^{\prime}$, then for any plan $y$, the manager prefers to realize a modification $y^{\prime}$ of $y$ rather than $y$ itself where $y^{\prime}$ takes the same values as $y$ except that $y^{\prime}$ takes the value $z$ whenever $y$ takes the value $z^{\prime}$. Since $y^{\prime} \leq y$, it is attainable whenever $y$ is attainable. But since such a plan $y^{\prime}$ is dominated, manager's choice will not be accepted by shareholders. The treatment of $z_{T} \rightarrow z_{T}-\phi_{T}\left(z_{0}, \ldots, z_{T}\right)$ is symmetric. The technicalities of the proof are due to 1 . the multiple dates setting, 2 . the fact that we have to deal with measurable modifications on nonzero measure sets, and 3 . the modifications should be made such that $C(y)=C\left(y^{\prime}\right)$.

In order to show that date- $T$ manager's compensation $\phi_{T}\left(z_{0}, \ldots, z_{T}\right)$ and the net production $z_{T} \rightarrow z_{T}-\phi_{T}\left(z_{0}, \ldots, z_{t-1}, z_{t}\right)$ are nondecreasing, we have to first provide a definition of monotonicity that is robust to the fact that the considered functions can be modified on a 0 measure set without modifying the behavior of the manager nor those of the shareholders.

Definition $A$ function $\varphi: \mathbb{R} \rightarrow \mathbb{R}$ is nondecreasing a.e. if

$$
\mu^{2}\left(\left\{\left(z, z^{\prime}\right) \in \mathbb{R}^{2}:\left(z-z^{\prime}\right)\left(\varphi(z)-\varphi\left(z^{\prime}\right)\right)<0\right\}\right)=0 .
$$

Let us show that, for almost all $\left(z_{0}, \ldots, z_{T-1}\right) \in \mathbb{R}_{+}^{T}$, both functions $z_{T} \rightarrow \phi_{T}\left(z_{0}, \ldots, z_{T}\right)$ and $z_{T} \rightarrow z_{T}-\phi_{T}\left(z_{0}, \ldots, z_{t}\right)$ are nondecreasing a.e. 
By definition of the conditional expectation, there exists $Q$ such that $E[\hat{q} \mid \hat{y}]=Q(\hat{y})$. Since $\hat{q} \gg 0, P$-a.e., we have $Q>0 \mu$-a.e. Furthermore, it is immediate that, for almost every $\left(z_{0}, \ldots, z_{T}\right) \in \mathbb{R}_{+}^{T+1}$, we have

$$
\begin{aligned}
& E\left[\hat{q}_{T} \mid\left(\hat{y}_{0}, \ldots, \hat{y}_{T-1}\right)=\left(z_{0}, \ldots, z_{T-1}\right) \text { and } \hat{y}_{T} \in\left[z_{T}-\varepsilon, z_{T}+\varepsilon\right)\right] \\
& =\frac{\int_{u \in\left[z_{T}-\varepsilon, z_{T}+\varepsilon\right)} h_{y}\left(z_{0}, \ldots, z_{T-1}, u\right) Q\left(z_{0}, \ldots, z_{T-1}, u\right) d u}{\int_{u \in\left[z_{T}-\varepsilon, z_{T}+\varepsilon\right)} h_{y}\left(z_{0}, \ldots, z_{T-1}, u\right) d u} \underset{\varepsilon \rightarrow 0}{\rightarrow} Q\left(z_{0}, \ldots, z_{T}\right) .
\end{aligned}
$$

Let us assume that $z_{T} \rightarrow \phi_{T}\left(z_{0}, \ldots, z_{T}\right)$ is not nondecreasing and, for each $\left(z_{0}, \ldots, z_{T-1}\right)$, let

$$
\begin{aligned}
& A\left(z_{0}, \ldots, z_{T-1}\right)= \\
& \left\{\left(z_{T}, z_{T}^{\prime}\right) \in \mathbb{R}_{+}^{2}:\left(z_{T}-z_{T}^{\prime}\right)\left(\phi_{T}\left(z_{0}, \ldots, z_{T-1}, z_{T}\right)-\phi_{T}\left(z_{0}, \ldots, z_{T-1}, z_{T}^{\prime}\right)\right)<0\right\} .
\end{aligned}
$$

We have, $\mu^{2}\left(A\left(z_{0}, \ldots, z_{T-1}\right)\right)>0$ for all $\left(z_{0}, \ldots, z_{T-1}\right) \in B$ for some Borelmeasurable set $B \subset \mathbb{R}_{+}^{T}$ with $\mu^{T}(B)>0$. We know that $\mu^{T+1}\left(\mathbb{R}_{+}^{T+1} \backslash C\right)=0$ where $C$ is the Borel-measurable set of continuity points of $\phi_{T}$ for which we further have $h_{y}>0$ and $Q>0$. Hence, we may modify $A$ and $B$ in order to have $\left(z_{0}, \ldots, z_{T}\right)$ and $\left(z_{0}, \ldots, z_{T}^{\prime}\right)$ in $C$ for all $\left(z_{0}, \ldots, z_{T-1}\right) \in B$ and $\left(z_{T}, z_{T}^{\prime}\right) \in A\left(z_{0}, \ldots, z_{T-1}\right)$.

For $\left(z_{0}, \ldots, z_{T-1}\right) \in B$, there exists then $(a, b) \in A\left(z_{0}, \ldots, z_{T-1}\right)$. We may assume $a<b$ and then

$$
\phi^{a} \stackrel{\text { def }}{=} \phi_{T}\left(z_{0}, \ldots, z_{T-1}, a\right)>\phi_{T}\left(z_{0}, \ldots, z_{T-1}, b\right) \stackrel{\text { def }}{=} \phi^{b} .
$$

For $\eta>0$, there exists $\varepsilon>0$ such that $\left|\phi_{T}\left(z_{0}, \ldots, z_{T-1}, z\right)-\phi^{a}\right|<\eta$ for $z \in I=[a-\varepsilon, a+\varepsilon)$ and $\left|\phi_{T}\left(z_{0}, \ldots, z_{T-1}, z\right)-\phi^{b}\right|<\eta$ for $z \in J=$ $[b-\varepsilon, b+\varepsilon)$. If $\eta$ and $\varepsilon$ are chosen such that $\eta<\frac{\phi^{a}-\phi^{b}}{2}$ and $\varepsilon<\frac{b-a}{2}$, we have $I<J$ and $\phi_{T}\left(z_{0}, \ldots, z_{T-1}, I\right)>\phi_{T}\left(z_{0}, \ldots, z_{T-1}, J\right)$. Since $I, J, a$ and $b$ depend on $z=\left(z_{0}, \ldots, z_{T-1}\right)$, we denote them by $I_{z}, J_{z}, a_{z}$ and $b_{z}$.

Let $\left(\alpha, \beta, \alpha^{\prime}, \beta^{\prime}\right)$ be rational numbers such that $\alpha<\beta<\alpha^{\prime}<\beta^{\prime}$ and let

$$
\begin{aligned}
& B\left(\alpha, \beta, \alpha^{\prime}, \beta^{\prime}\right)= \\
& \left\{z \in B: \alpha \in\left[a_{z}-\varepsilon, a_{z}\right), \beta \in\left[a_{z}, a_{z}+\varepsilon\right), \alpha^{\prime} \in\left[b_{z}-\varepsilon, b_{z}\right), \beta^{\prime} \in\left[b_{z}, b_{z}+\varepsilon\right)\right\} .
\end{aligned}
$$


It is easy to check that $B=\cup_{\alpha, \beta, \alpha^{\prime}, \beta^{\prime}} B\left(\alpha, \beta, \alpha^{\prime}, \beta^{\prime}\right)$. As the set of $\left(\alpha, \beta, \alpha^{\prime}, \beta^{\prime}\right)$ is countable, there exists $\left(\bar{\alpha}, \bar{\beta}, \bar{\alpha}^{\prime}, \bar{\beta}^{\prime}\right)$ such that $\mu^{T-1}\left(B\left(\bar{\alpha}, \bar{\beta}, \bar{\alpha}^{\prime}, \bar{\beta}^{\prime}\right)\right)>0$. Let $a$ and $b$ and $\varepsilon$ be sufficiently small so that we further have $[a-\varepsilon, a+\varepsilon) \subset$ $[\bar{\alpha}, \bar{\beta})$ and $[b-\varepsilon, b+\varepsilon) \subset\left[\bar{\alpha}^{\prime}, \bar{\beta}^{\prime}\right)$. From now on, we denote by $B^{\prime}$ the set $B\left(\bar{\alpha}, \bar{\beta}, \bar{\alpha}^{\prime}, \bar{\beta}^{\prime}\right)$, by $I^{\prime}$ the interval $[a-\varepsilon, a+\varepsilon)$ and by $J^{\prime}$ the interval $[b-\varepsilon, b+\varepsilon)$. By considering a possibly smaller $B^{\prime}$ of positive measure, we may assume (without loss of generality) that $\phi_{T}$ is continuous at $\left(z_{0}, \ldots, z_{T-1}, a\right)$ and at $\left(z_{0}, \ldots, z_{T-1}, b\right)$ for all $\left(z_{0}, \ldots, z_{T-1}\right) \in B^{\prime}$.

The intervals $I^{\prime}$ and $J^{\prime}$ do not depend on $\left(z_{0}, \ldots, z_{T-1}\right) \in B$ and we have $I^{\prime}<J^{\prime}$ and $\phi_{T}\left(z_{0}, \ldots, z_{T-1}, I^{\prime}\right)>\phi_{T}\left(z_{0}, \ldots, z_{T-1}, J^{\prime}\right)$ for all $\left(z_{0}, \ldots, z_{T-1}\right) \in$ $B^{\prime}$.

Let $f: \mathbb{R} \rightarrow \mathbb{R} \backslash J^{\prime}$ be defined by $f(z)=\frac{1}{2}(a-\varepsilon)+\frac{1}{2} z$ on $I^{\prime}, f(z)=$ $a+\frac{1}{2}(z-b+\varepsilon)$ on $J^{\prime}$ and $f(z)=z$ elsewhere. We have $f(z) \leq z$ for all $z$ and $f$ admits an inverse denoted by $g$.

Let the process $\tilde{y}$ be defined by $\left(\tilde{y}_{1}, \ldots, \tilde{y}_{T}\right)=\left(\hat{y}_{1}, \ldots, \hat{y}_{T-1}, f\left(\hat{y}_{T}\right)\right)$ when $\left(\hat{y}_{1}, \ldots, \hat{y}_{T-1}\right) \in B^{\prime}$ and $\left(\tilde{y}_{1}, \ldots, \tilde{y}_{T}\right)=\left(\hat{y}_{1}, \ldots, \hat{y}_{T-1}, \hat{y}_{T}\right)$ elsewhere. As $\tilde{y} \leq$ $\hat{y}, \tilde{y} \in Y$. By definition of $g$, we have $\left(\hat{y}_{1}, \ldots, \hat{y}_{T-1}, \hat{y}_{T}\right)=\left(\tilde{y}_{1}, \ldots, \tilde{y}_{T-1}, g\left(\tilde{y}_{T}\right)\right)$ when $\left(\tilde{y}_{1}, \ldots, \tilde{y}_{T-1}\right) \in B^{\prime}$ and $\left(\hat{y}_{1}, \ldots, \hat{y}_{T}\right)=\left(\tilde{y}_{1}, \ldots, \tilde{y}_{T}\right)$ elsewhere. Hence $\hat{y}$ and $\tilde{y}$ generate then the same information structure and $\mathcal{C}(\tilde{y})=\mathcal{C}(\hat{y})$.

Observe that for $\left(\hat{y}_{1}, \ldots, \hat{y}_{T-1}\right) \in B^{\prime}$, if $\hat{y}_{T} \in I^{\prime}$, then $\tilde{y}_{T} \in I^{\prime}$ and

$$
\left|\phi_{T}\left(\hat{y}_{1}, \ldots, \hat{y}_{T}\right)-\phi_{T}\left(\tilde{y}_{1}, \ldots, \tilde{y}_{T}\right)\right|<2 \eta
$$

if $\hat{y}_{T} \in J^{\prime}$, then $\tilde{y}_{T} \in I^{\prime}$ and $\phi_{T}\left(\hat{y}_{1}, \ldots, \hat{y}_{T}\right)-\phi\left(\tilde{y}_{1}, \ldots, \tilde{y}_{T}\right)<\phi(b)-\phi(a)+2 \eta$ and, we have $\phi(\hat{y})=\phi(\tilde{y})$ elsewhere. Therefore, for $\left(z_{0}, \ldots, z_{T-1}\right) \in B^{\prime}$, we have

$$
\begin{aligned}
& \frac{E_{\left(z_{0}, \ldots, z_{T-1}\right)}\left[\hat{q}_{T} \phi_{T}(\hat{y})-\hat{q}_{T} \phi_{T}(\tilde{y})\right]}{2 \varepsilon} \\
& <\frac{2 \eta P_{\left(z_{0}, \ldots, z_{T-1}\right)}\left(\hat{y}_{T} \in I^{\prime} \cup J^{\prime}\right) E_{\left(z_{0}, \ldots, z_{T-1}\right)}\left[\hat{q}_{T} \mid \hat{y}_{T} \in I^{\prime} \cup J^{\prime}\right]}{2 \varepsilon} \\
& +\frac{2\left(\phi_{T}\left(z_{0}, \ldots, z_{T-1}, b\right)-\phi_{T}\left(z_{0}, \ldots, z_{T-1}, a\right)\right) P_{\left(z_{0}, \ldots, z_{T-1}\right)}\left(\hat{y}_{T} \in J\right) E_{\left(z_{0}, \ldots, z_{T-1}\right)}\left[\hat{q}_{T} \mid \hat{y}_{T} \in J\right]}{2 \varepsilon} \\
& \underset{\varepsilon \rightarrow 0}{\rightarrow} 2 \eta\left(h\left(z_{0}, \ldots, z_{T-1}, a\right)+h\left(z_{0}, \ldots, z_{T-1}, b\right)\right) Q\left(z_{0}, \ldots, z_{T-1} a\right) \\
& +\left(\phi_{T}\left(z_{0}, \ldots, z_{T-1}, b\right)-\phi_{T}\left(z_{0}, \ldots, z_{T-1}, a\right)\right) h\left(z_{0}, \ldots, z_{T-1}, b\right) Q\left(z_{0}, \ldots, z_{T-1} b\right)
\end{aligned}
$$


where $E_{\left(z_{0}, \ldots, z_{T-1}\right)}$ and $P_{\left(z_{0}, \ldots, z_{T-1}\right)}$ correspond, respectively, to the expectation and to the probability conditional to $\left(\hat{y}_{1}, \ldots, \hat{y}_{T-1}\right)=\left(z_{0}, \ldots, z_{T-1}\right)$. Since $\phi_{T}\left(z_{0}, \ldots, z_{T-1}, b\right)-\phi_{T}\left(z_{0}, \ldots, z_{T-1}, a\right)<0$, for $\eta$ and $\varepsilon$ sufficiently small and $\left(z_{0}, \ldots, z_{T-1}\right) \in B^{\prime}$, we have $\frac{E_{\left(z_{0}, \ldots, z_{T-1}\right)}\left(\hat{q}_{T} \phi_{T}(\hat{y})-\hat{q}_{T} \phi_{T}(\tilde{y})\right]}{2 \varepsilon}<0$. For $\left(z_{0}, \ldots, z_{T-1}\right) \notin B^{\prime}$ and $\left(\hat{y}_{1}, \ldots, \hat{y}_{T-1}\right)=\left(z_{0}, \ldots, z_{T-1}\right)$, we have $\hat{y}=\tilde{y}$ and $\frac{E_{\left(z_{0}, \ldots, z_{T-1}\right)}\left(\hat{q}_{T} \phi_{T}(\hat{y})-\hat{q}_{T} \phi_{T}(\tilde{y})\right]}{2 \varepsilon}=0$. Since $\mu^{T-1}\left(B^{\prime}\right)>0$, by the law of iterated expectations, we obtain $E\left[\hat{q}_{T} \phi_{T}(\hat{y})\right]<E\left[\hat{q}_{T} \phi_{T}(\tilde{y})\right]$ and $\hat{q} \cdot \Phi(\hat{y})<\hat{q} \cdot \Phi(\tilde{y})$.

Since $\mathcal{C}(\tilde{y})=\mathcal{C}(\hat{y})$, we have $V^{m}(\hat{q}, \tilde{y})>V^{m}(\hat{q}, \hat{y})$ which is impossible. The function $I d-\phi_{T}$ is treated similarly, using $V^{i}$ instead of $V^{m}$.

Step 2. Differentiability

Since $z_{T} \rightarrow \phi_{T}\left(z_{1}, \ldots, z_{T}\right)$ and $z_{T} \rightarrow z_{T}-\phi_{T}\left(z_{1}, \ldots, z_{T}\right)$ are nondecreasing then $z_{T} \rightarrow \phi_{T}\left(z_{1}, \ldots, z_{T}\right)$ is 1-Lipshitz and then differentiable a.e.

SteP 3. Linearity at date $T$

Let us show that $\hat{q} \cdot \Phi(\hat{y})=\max _{Y(\hat{y}, T-1)} \hat{q} \cdot \Phi(y)$. Assume that there exists $y^{\prime} \in Y(\hat{y}, T-1)_{+}$such that $\hat{q} \cdot \Phi\left(y^{\prime}\right)>\hat{q} \cdot \Phi(\hat{y})$. By Lemma 11 and by continuity of $\phi_{T}$, there exists $y^{\prime \prime} \in Y(\hat{y}, T-1)$ close to $y^{\prime}$ such that $\hat{q} \cdot \Phi\left(y^{\prime \prime}\right)>\hat{q} \cdot \Phi(\hat{y})$ and $\mathcal{C}(\hat{y}) \subset \mathcal{C}\left(y^{\prime \prime}\right)$. From the definition of the indirect utility, we have $V_{m}\left(y^{\prime \prime}, \hat{q}\right)>V_{m}(\hat{y}, \hat{q})$ which violates assertion 4 of the definition of equilibrium. We have then $\hat{q} \cdot \Phi(\hat{y})=\max _{Y(\hat{y}, T-1)} \hat{q} \cdot \Phi(y)$. From Proposition 1, $N_{Y}(\hat{y})=\{\alpha \hat{q}, \alpha>0\}$ and by Assumption P4, we have then $N_{Y(\hat{y}, T-1)}(y)=\left\{\left(q_{0}, \ldots, q_{T-1}, \alpha \hat{q}_{T}\right): \alpha>0\right\}$. As $\phi_{T}$ is differentiable a.e., from the first order conditions, there exists $\alpha>0$ such that,

$$
\hat{q}_{T} \frac{\partial \phi_{T}}{\partial y_{T}}\left(\hat{y}_{0}, \ldots, \hat{y}_{T}\right)=\alpha \hat{q}_{T}
$$

As $\hat{q}_{T} \gg 0$ and as from assumption $\mathrm{P}, \hat{y}$ takes all possible values in $(0, \infty)^{T}$, we have

$$
\frac{\partial \phi_{T}}{\partial x_{T}}\left(z_{0}, \ldots, z_{T}\right)=\alpha, \forall z \in \mathbb{R}_{+}^{T+1} .
$$

Integrating with respect to $z_{T}$, we get that for some functions $H: \mathbb{R}_{+}^{T} \rightarrow \mathbb{R}$

$$
\phi_{T}\left(z_{0}, \ldots, z_{T}\right)=\alpha z_{T}+H\left(z_{0}, \ldots, z_{T-1}\right) .
$$

Since $\phi_{T}\left(z_{0}, \ldots, z_{T}\right) \leq z_{T}, \phi_{T}\left(z_{0}, \ldots, z_{T-1}, 0\right)=0$. Setting $z_{T}=0$ in equation (15), we obtain that $H=0$ and $\phi_{T}\left(z_{0}, \ldots, z_{T}\right)=\alpha z_{T}$.

STEP 4. Backward induction 
The first step above relies, in particular, on the fact that $z_{T}$ only intervenes in $\phi_{T}$ and not in $\phi_{t}$ for $t \neq T$. Due to Step 3, we know now that $z_{T-1}$ does not intervene in $\phi_{T}$ and hence only intervenes in $\phi_{T-1}$. We may then apply the above methodology to prove that $\phi_{T-1}$ is nondecreasing and then differentiable a.e. Similarly, the first order condition with respect to $z_{T-1}$ only involves $\phi_{T-1}$ and is similar to 14 replacing $T$ by $T-1$. We obtain that $\phi_{T-1}\left(z_{0}, \ldots, z_{T-1}\right)=\alpha z_{T-1}$ and by backward induction, we obtain that $\phi_{t}\left(z_{0}, \ldots, z_{t}\right)=\alpha z_{t}$ for all $t$ and all $\left(z_{0}, \ldots, z_{t}\right) \in \mathbb{R}_{+}^{t+1}$. As $0 \leq \Phi(y) \leq y, 0 \leq \alpha \leq 1$.

STEP $5.0<\alpha<1$

Note that $\alpha=0$ leads to $V^{m}(y, \hat{q})$ independent of $y$ which is not compatible with the 4 th equilibrium condition. Similarly, $\alpha=1$ leads to $V^{i}(y, \hat{q})$ independent of $y$ which is not compatible with the 6th equilibrium condition.

Proof of Theorem 3. Let $\left(\Phi,\left(\hat{c}^{i}\right)_{i}, \hat{c}_{m}, \hat{q}, \hat{y}\right)$ be an equilibrium. From Theorem 2, $\Phi=\Phi^{\alpha}$ for some $\alpha$ s.t. $0<\alpha<1$. Let us first show that $V_{m}(\hat{y}, \hat{q})=U_{m}\left(\hat{c}_{m}\right)=\max \left\{U_{m}(c), \hat{q} \cdot c \leq \hat{q} \cdot \Phi(\hat{y})\right\}$ or, in other words, that we can relax the condition $c \in \mathcal{C}(\hat{y})$. If this is not the case, there exists $c$ such that $\hat{q} \cdot c \leq \hat{q} \cdot \Phi(\hat{y})$ and $U_{m}(c)>U_{m}\left(\hat{c}_{m}\right)$. As $U_{m}$ is homogeneous, for $\epsilon$ small enough, we have $\hat{q} \cdot(1-\epsilon) c<\hat{q} \cdot \Phi(\hat{y})$ and $U_{m}((1-\epsilon) c)>U_{m}\left(\hat{c}_{m}\right)$. By continuity of $q \cdot \Phi$, let $\varepsilon>0$ such that $|\hat{q} \cdot \Phi(\hat{y})-\hat{q} \cdot \Phi(y)|<\hat{q} \cdot \Phi(\hat{y})-$ $\hat{q} \cdot(1-\epsilon) c$ for all $y$ such that $\|y-\hat{y}\|<\varepsilon$. By Lemma 11, there exists $y \in X_{+}$such that $y<<\hat{y},\|y-\hat{y}\|<\varepsilon$ and $C((1-\epsilon) c) \subset C(y)$. We have then $y \in Y_{+},(1-\epsilon) c \subset C(y)$ and $\hat{q} \cdot(1-\epsilon) c<\hat{q} \cdot \Phi(y)$ which gives $V_{m}(y, \hat{q}) \geq U_{m}((1-\epsilon) c)>U_{m}\left(\hat{c}_{m}\right)=V_{m}(\hat{y}, \hat{q})$ which contradicts Condition 4 in the definition of a manager-shareholders equilibrium.

Therefore, there exists $\lambda>0$ such that

$$
\alpha \exp \left(-\rho_{m} t\right) M_{m, t} u^{\prime}\left(\hat{c}_{m, t}\right)=\lambda \hat{q}_{t}, \text { for all } t
$$

Furthermore from the 5th equilibrium condition,

$$
\max _{y \in Y} \sum_{t=0}^{T} \exp \left(-\rho_{m} t\right) E\left[M_{m, t} u\left(\hat{C}_{m, t}\left(y_{0}, \ldots, y_{t}\right)\right]=U_{m}\left(\hat{C}_{m}(\hat{y})\right)\right.
$$


Let us show that $\hat{C}_{m, T}\left(z_{0}, \ldots, z_{T-1}, z_{T}\right)$ is nondecreasing in $z_{T}$. The proof is similar to the proof of Step 1 in Theorem 2 and we only provide a sketch of it. Let $\left(z_{0}, \ldots, z_{T-1}\right) \in \mathbb{R}_{+}^{T}$ be given and let us define $\tilde{C}\left(z_{T}\right)$ by $\tilde{C}\left(z_{T}\right) \stackrel{\text { def }}{=} \hat{C}_{m, T}\left(z_{0}, \ldots, z_{T-1}, z_{T}\right)$.

Let $A=\left\{\left(z, z^{\prime}\right) \in \mathbb{R}_{+}^{2}:\left(z-z^{\prime}\right)\left(\tilde{C}(z)-\tilde{C}\left(z^{\prime}\right)\right)<0\right\}$ and let us assume that $\mu \otimes \mu(A)>0$. By Fubini, there exists $z$ such that $\mu(B)>0$ with $B=$ $\left.\left\{z^{\prime}>z: \tilde{C}(z)>\tilde{C}\left(z^{\prime}\right)\right)\right\}$. Let us consider $\hat{y}^{\prime}$ such that $\hat{y}^{\prime}=\left(z_{0}, \ldots, z_{T-1}, z\right)$ when $\hat{y}=\left(z_{0}, \ldots, z_{T-1}, z_{T}^{\prime}\right)$ with $z_{T}^{\prime} \in B$ and $\hat{y}^{\prime}=\hat{y}$ elsewhere. We have $\hat{y}^{\prime} \leq \hat{y}$ and then $\hat{y}^{\prime} \in Y$ and $\hat{C}_{m, T}\left(\hat{y}^{\prime}\right)>\hat{C}_{m, T}(\hat{y})$. If we were able do that for a set of $\left(z_{0}, \ldots, z_{T-1}\right)$ with positive measure then we would obtain a contradiction with the fact that $\hat{y}$ maximizes $U_{m}\left(\hat{C}_{m}(\hat{y})\right)$ on $Y$. Hence, for almost all $\left(z_{0}, \ldots, z_{T-1}\right) \in \mathbb{R}_{+}^{T}$, we have $\mu \otimes \mu(A)=0$ which means that $\hat{C}_{m, T}\left(z_{0}, \ldots, z_{T-1}, z_{T}\right)$ is nondecreasing a.e. in $z_{T}$ for almost all $\left(z_{0}, \ldots, z_{T-1}\right) \in \mathbb{R}_{+}^{T}$ and $\hat{C}_{m, T}\left(z_{0}, \ldots, z_{T-1}, z_{T}\right)$ admits a partial derivative with respect to $z_{T}$ almost everywhere.

From the first order conditions and Proposition 1 item 1, there exists $\nu>0$ such that

$$
\sum_{s=t}^{T} E_{t}\left[\frac{\partial \hat{C}_{m, s}}{\partial y_{t}}\left(\hat{y}_{0}, \ldots, \hat{y}_{s}\right) \exp \left(-\rho_{m} s\right) M_{m, s} u^{\prime}\left(\hat{c}_{m, s}\right)\right]=\nu \hat{q}_{t} \text { for all } t
$$

From Equations (16 ) and (17), we thus obtain that for all $t$ :

$$
\sum_{s=t}^{T} E_{t}\left[\frac{\partial\left(\hat{C}_{m, s}\right)}{\partial y_{t}}\left(\hat{y}_{0}, \ldots, \hat{y}_{s}\right) \exp \left(-\rho_{m} s\right) M_{m, s} u^{\prime}\left(\hat{c}_{m, s}\right)\right]=a \exp \left(-\rho_{m} t\right) M_{m, t} u^{\prime}\left(\hat{c}_{m, t}\right)
$$

for some $a>0$. In particular, at date $T$,

$$
\frac{\partial \hat{C}_{m, T}}{\partial y_{T}}\left(\hat{y}_{0}, \ldots, \hat{y}_{T}\right)=a \text {. }
$$

As $\hat{y}$ takes all possible values in $\mathbb{R}_{+}^{T+1}$, we have

$$
\frac{\partial \hat{C}_{m, T}}{\partial x_{T}}\left(z_{0}, \ldots, z_{T}\right)=a, \forall z \in \mathbb{R}_{+}^{T+1}
$$


Integrating with respect to $z_{T}$, we obtain

$$
\hat{C}_{m, T}\left(z_{0}, \ldots, z_{T}\right)=a z_{T}+H\left(z_{0}, \ldots, z_{T-1}\right)
$$

for some functions $H: \mathbb{R}_{+}^{T} \rightarrow \mathbb{R}$. Since $\hat{C}_{m, T}\left(z_{0}, \ldots, z_{T}\right) \leq z_{T}$, if $z_{T}=0$ $\hat{C}_{m, T}\left(z_{0}, \ldots, z_{T}\right)=0$. Setting $z_{T}=0$ in the previous equation, we obtain that $H=0$ and

$$
\hat{C}_{m, T}\left(z_{0}, \ldots, z_{T}\right)=a z_{T}, \forall\left(z_{0}, \ldots, z_{T}\right) \in \mathbb{R}_{+}^{T+1}
$$

Similarly, considering next Equation (18) at $t=T-1$, we obtain

$$
\hat{C}_{m, T-1}\left(z_{0}, \ldots, z_{T-1}\right)=a z_{T-1}
$$

By backward induction, we obtain that $\hat{C}_{m, t}\left(z_{0}, \ldots, z_{t}\right)=a z_{t}$ for all $t$ and all $\left(z_{0}, \ldots, z_{t}\right) \in \mathbb{R}_{+}^{t+1}$, and using the equality $\hat{q} \cdot \hat{C}_{m}(\hat{y})=\hat{q} \cdot \alpha \hat{y}$, that $a=\alpha$ or $\hat{C}_{m}=\Phi^{\alpha}$. Therefore, irrespective of the production process and of the history, the consumption of the manager at $t$ coincides with her compensation at $t$; that is, the manager does not trade in the financial market.

Proof of Theorem 4. The proof of assertion 1 may be found in Appendix 2. To prove assertion 2, from assertion 1, there exists a unique equilibrium associated to $(1-\alpha) Y$. From the homogeneity of preferences, one directly checks that $\left.\left((1-\alpha) c^{* i}\right)_{i}, q^{*},(1-\alpha) y^{*}\right)$ solves the three properties of a production equilibrium associated to $(1-\alpha) Y$.

Proof of Theorem 5. Let $0<\alpha<1$ be given and $\left(\Phi^{\alpha},\left(\hat{c}^{i}\right)_{i}, \alpha \hat{y}, \hat{q}, \hat{y}\right)$ be a manager-shareholders equilibrium. To prove assertion 1 , from assertion 2 of Definition 1 and from Theorems 2 and 3, we have, for some $\alpha$ such that $0<\alpha<1$,

$$
V_{m}(\hat{y}, \hat{q})=U_{m}\left(\hat{C}_{m}(\hat{y})\right)=U_{m}(\alpha \hat{y}) .
$$

As $V_{m}(y, \hat{q}) \geq U_{m}(\alpha y)$ for all $y \in Y$, from assertion 4 of Definition 1, we have

$$
V_{m}(\hat{y}, \hat{q})=U_{m}(\alpha \hat{y}) \geq V_{m}(y, \hat{q}) \geq U_{m}(\alpha y), \forall y \in Y,
$$

proving the desired assertion. To prove assertion 2, from assertion 1 of Definition 1 and Assumption (P5), $\hat{c}^{i}$ maximizes $U^{i}(c)$ s.t. $\hat{q} \cdot c \leq \nu^{i}(\hat{q} \cdot$ 
$((1-\alpha) \hat{y}))$, hence the first item of the definition of a production equilibrium associated to $(1-\alpha) Y$ is verified. From Proposition $1, \hat{q} \cdot(1-\alpha) \hat{y} \geq$ $\hat{q} \cdot(1-\alpha) y$ for all $y \in Y$. Lastly from Theorems 2 and 3 and assertion 3 of the definition of a production equilibrium, $\sum_{i} \hat{c}^{i}=(1-\alpha) \hat{y}$. Therefore $\left(\left(\hat{c}^{i}\right)_{i}, \hat{q},(1-\alpha) \hat{y}\right)$ is the production equilibrium associated to $(1-\alpha) Y$ and from Theorem $4, \hat{c}^{i}=(1-\alpha) c^{* i}$ for all $i, \hat{q}=\nu q^{*}$ for some $\nu>0$ and $\hat{y}=y^{*}$.

Conversely, let us assume that Assertions 1 and 2 of Theorem 5 are satisfied. By definition of the production equilibrium, the plan $c^{* i}$ maximizes $U^{i}$ under the budget constraint $\hat{q} \cdot c \leq \nu^{i} \hat{q} \cdot y^{*}$ hence $\hat{c}^{i}=(1-\alpha) c^{* i}$ maximizes $U^{i}$ under the budget constraint $\hat{q} \cdot c \leq \nu^{i} \hat{q} \cdot(1-\alpha) y^{*}=\nu^{i} \hat{q} \cdot\left(\hat{y}-\Phi^{\alpha}(\hat{y})\right)$. Furthermore, by Assumption P5, $C(\hat{y})=X$. Then, Condition 1 in Definition 1 is satisfied. The same reasoning applies to Condition 2. By definition of the production equilibrium, we have $\sum_{i} \hat{c}^{i}=(1-\alpha) \hat{y}$ which immediately gives Condition 3. Assertion 1 of Theorem 5 is equivalent to Condition 5 . Let $y \in Y$, by definition of the production equilibrium, we have $\hat{q} \cdot y \leq \hat{q} \cdot \hat{y}$. If $\hat{q} \cdot y=\hat{q} \cdot \hat{y}$, the strict convexity from above gives the existence of $y^{\prime}$ in $Y$ such that $y^{\prime}>\frac{1}{2} y+\frac{1}{2} \hat{y}$ and, since $q \gg 0, \hat{q} \cdot y^{\prime}>\hat{q} \cdot \hat{y}$ which contradicts the definition of the production equilibrium. We have then $\hat{q} \cdot y<\hat{q} \cdot \hat{y}$ and since $C(\hat{y})=X$, by strict monotonicity of the utility function, we have $V_{m}(\hat{y}, \hat{q})>V_{m}(y, \hat{q})$. Therefore, Condition 4 is satisfied. Condition 6 is derived similarly.

Proof of Theorem 6. To prove assertion 1, from Theorem 4, there exists a unique production equilibrium associated to $Y$ denoted by $\left(\left(c^{* i}\right)_{i}, q^{*}, y^{*}\right)$ with $q^{*}$ defined up to a multiplicative constant. The vector of equilibrium utility weights $\left(\lambda^{* i}\right)_{i}$ in $\Lambda$ and the scaling constant $\nu>0$ are uniquely defined by the shareholder's first order conditions at equilibrium given by (7). To prove assertion 2, from Equation (21), the representative shareholder's utility at equilibrium is $N\left(\lambda^{*}\right) \cdot u\left(y^{*}\right)$. Let us define $\left(\tilde{\rho}_{t}\right)$ and $\left(\tilde{M}_{t}\right)$ by induction as follows

$$
\frac{\exp \left(\tilde{\rho}_{t+1}(t+1)\right)}{\exp \left(\tilde{\rho}_{t} t\right)}=\frac{N_{t}}{E_{t}\left[N_{t+1}\left(\lambda^{*}\right)\right]} \text { and } \tilde{M}_{t+1}=N_{t+1}\left(\lambda^{*}\right) \exp \left(\tilde{\rho}_{t+1}(t+1)\right)
$$

The process $\left(\exp \left(-\tilde{\rho}_{t} t\right)\right)$ is predictable and $\left(\tilde{M}_{t}\right)$ is a martingale and we have $\exp \left(-\tilde{\rho}_{t} t\right) \tilde{M}_{t}=N_{t}\left(\lambda^{*}\right)$. Furthermore, this decomposition is unique. 
Indeed, if $\exp \left(-\tilde{\rho}_{t}^{1} t\right) \tilde{M}_{t}^{1}=\exp \left(-\tilde{\rho}_{t}^{2} t\right) \tilde{M}_{t}^{2}$, then taking the expectation for $t=1$, we obtain $\tilde{\rho}_{1}^{1}=\tilde{\rho}_{1}^{2}$ and from there $\tilde{M}_{1}^{1}=\tilde{M}_{2}^{2}$. If we take now the expectation for $t=2$ conditional to date 1 , we have

$$
\begin{aligned}
\exp \left(-2 \tilde{\rho}_{2}^{1}\right) \tilde{M}_{1}^{1} & =E_{1}\left[\exp \left(-2 \tilde{\rho}_{2}^{1}\right) \tilde{M}_{2}^{1}\right]=E_{1}\left[\exp \left(-2 \tilde{\rho}_{2}^{2}\right) \tilde{M}_{2}^{2}\right] \\
& =\exp \left(-2 \tilde{\rho}_{2}^{2}\right) E_{1}\left[\tilde{M}_{2}^{2}\right]=\exp \left(-2 \tilde{\rho}_{2}^{2}\right) \tilde{M}_{1}^{2}
\end{aligned}
$$

and since $\tilde{M}_{1}^{1}=\tilde{M}_{1}^{2}$, we obtain $\tilde{\rho}_{2}^{1}=\tilde{\rho}_{2}^{2}$ and from there $\tilde{M}_{2}^{1}=\tilde{M}_{2}^{2}$. By induction, we get $\tilde{M}^{1}=\tilde{M}^{2}$ and $\tilde{\rho}^{1}=\tilde{\rho}^{2}$.

To prove the last assertion, at the production equilibrium associated to $(1-\alpha) Y$, the utility weights and $\nu_{\alpha}>0$ are defined by the first order conditions $\lambda^{* i} M_{t}^{i} \exp \left(-\rho^{i} t\right) u^{\prime}\left((1-\alpha) c_{t}^{* i}\right)=\nu_{\alpha} q_{t}^{*}$ for all $t$ and all $i$ which defines the same equilibrium utility weights in $\Lambda$, hence the same $N\left(\lambda^{*}\right)$ and the same representative shareholder utility.

Proof of Theorem 7. From Theorem 5, the manager maximizes $U_{m}(\alpha y)$ over $Y$. Therefore she chooses $y^{*}$ if and only if the process

$$
\left(\exp \left(-\rho_{m} t\right) M_{m, t} u^{\prime}\left(\alpha y^{*}\right)_{t \in \mathbb{T}} \in N_{Y}\left(y^{*}\right)=\left\{\nu q^{*}, \nu>0\right\}\right.
$$

Moreover $q^{*}$ is proportional to the marginal utility of the representative shareholder at equilibrium. Using the homogeneity of $u$, we must have, for some $\nu>0$ and for every $t$

$$
\left(\exp \left(-\rho_{m} t\right) M_{m, t} u^{\prime}\left(y_{t}^{*}\right)=\nu \exp \left(-\tilde{\rho}_{t} t\right) \tilde{M}_{t} u^{\prime}\left(y_{t}^{*}\right)\right.
$$

Cancelling $u^{\prime}\left(y_{t}^{*}\right)$, on both sides and setting $t=0$, we obtain $\nu=1$ and (9).

Since $M_{m, t}$ and $\tilde{M}_{t}$ are martingales and $\tilde{\rho}_{t}$ is predictable, we have $\rho_{m}=$ $\tilde{\rho}_{t}$ for all $t$ and $M_{m}=\tilde{M}$ by the uniqueness of such a decomposition as proven in Theorem 6.

Proof of Propositions 8 and 9. The proof of Proposition 8 may be found in Subsection 5.2. and that of Proposition 9 in Subsection 5.3.

Proof of $E_{t}\left[\left(\sum_{i}\left(\lambda^{* i} M_{t+1}^{i}\right)^{\frac{1}{1-\gamma}}\right)^{1-\gamma}\right] \leq\left(\sum_{i}\left(\lambda^{* i} M_{t}^{i}\right)^{\frac{1}{1-\gamma}}\right)^{1-\gamma}$. 
Let $B \in F_{t}$, we have

$$
\begin{aligned}
E\left[\left(\sum_{i}\left(\lambda^{* i} M_{t+1}^{i}\right)^{\frac{1}{1-\gamma}}\right)^{1-\gamma} 1_{B}\right] & =\left(\left\|\sum_{i}\left(\lambda^{* i} M_{t+1}^{i}\right)^{\frac{1}{1-\gamma}} 1_{B}\right\|_{1-\gamma}\right)^{1-\gamma} \\
& \leq\left(\sum_{i}\left\|\left(\lambda^{* i} M_{t+1}^{i}\right)^{\frac{1}{1-\gamma}} 1_{B}\right\|_{1-\gamma}\right)^{1-\gamma} \\
& \leq\left(\sum_{i}\left(E\left[\lambda^{* i} M_{t}^{i} 1_{B}\right]\right)^{\frac{1}{1-\gamma}}\right)^{1-\gamma}
\end{aligned}
$$

which gives

$$
E_{t}\left[\left(\sum_{i}\left(\lambda^{* i} M_{t+1}^{i}\right)^{\frac{1}{1-\gamma}}\right)^{1-\gamma}\right] \leq\left(\sum_{i}\left(\lambda^{* i} M_{t}^{i}\right)^{\frac{1}{1-\gamma}}\right)^{1-\gamma} .
$$

Lemma 12 Let $0<a_{1}<\ldots<a_{N}$ and $0<b_{1}<\ldots<b_{N}$, the matrix $\left(a_{i}^{b_{j}}\right)$ is invertible.

Proof of the Lemma. Let $\left(\lambda_{1}, \ldots, \lambda_{N}\right)$ such that $\sum_{j=1}^{N} \lambda_{j} a_{i}^{b_{j}}=0$ for all $i$ and let $f$ defined by $f(x)=\sum_{j=1}^{N} \lambda_{j} x^{b_{j}}$. We have $f\left(a_{i}\right)=0$ for $i=1, \ldots, N$, and $g\left(a_{i}\right)=0$ for $i=1, \ldots, N$ with $g(x)=\sum_{j=1}^{N} \lambda_{j} x^{b_{j}-b_{1}}$. Therefore, there exists $\left(a_{1}^{1}, \ldots, a_{N-1}^{1}\right)$ such that $a_{i}^{1} \in\left(a_{i}, a_{i+1}\right)$ and $g^{\prime}\left(a_{i}^{1}\right)=0$ for $i=1, \ldots, N-1$. We have $g^{\prime}(x)=\sum_{j=2}^{N} \lambda_{j}\left(b_{j}-b_{1}\right) x^{b_{j}-b_{1}-1}$ and then $g^{1}\left(a_{i}^{1}\right)=0$ for $i=1, \ldots, N-1$ with $g^{1}(x)=\sum_{j=2}^{N} \lambda_{j}\left(b_{j}-b_{1}\right) x^{b_{j}-b_{2}}$. Therefore, there exists $\left(a_{1}^{2}, \ldots, a_{N-2}^{2}\right)$ such that $a_{i}^{2} \in\left(a_{i}^{1}, a_{i+1}^{1}\right)$ and $\left(g^{1}\right)^{\prime}\left(a_{i}^{2}\right)=0$ for $i=1, \ldots, N-2$ or $g^{2}\left(a_{i}^{2}\right)=0$ for $i=1, \ldots, N-2$ with $g^{2}(x)=\sum_{j=3}^{N} \lambda_{j}\left(b_{j}-\right.$ $\left.b_{1}\right)\left(b_{j}-b_{2}\right) x^{b_{j}-b_{3}}$. By iteration, we have $a_{1}^{N-1}>g^{N-1}\left(a_{1}^{N-1}\right)=0$ with $\left.g^{N-1}(x)=\sum_{j=N}^{N} \lambda_{j}\left(b_{j}-b_{1}\right)\left(b_{j}-b_{2}\right) \ldots\left(b_{j}-b_{N-1}\right)\right) x^{b_{j}-b_{N}}$ which gives $\lambda_{N}\left(b_{N}-\right.$ $\left.b_{1}\right)\left(b_{N}-b_{2}\right) \ldots\left(b_{N}-b_{n-1}\right)=0$ or $\lambda_{n}=0$. By iteration, we obtain $\lambda_{N-1}=0$ and then $\lambda_{j}=0$ for $j=1, \ldots, N$.

Proof of Proposition 10. When dealing with equation (13), if two shareholders $i$ and $j$ have the same time discount rate, we may replace them by a single shareholder with the same discount rate, the belief $\frac{\lambda^{* i} M^{i}+\lambda^{* j} M^{j}}{\lambda^{* i}+\lambda^{* j}}$ and a weight $\lambda^{* i}+\lambda^{* j}$. Hence, we will assume without loss of generality that $\rho^{i} \neq \rho^{j}$ for all $(i, j)$ with $i \neq j$. We further assume that $\rho_{1}>\ldots>\rho_{N}$. Since there are at least $N$ dates $t \in \mathbb{T}$ such that $t>s$, let $\left(t_{1}, \ldots, t_{N}\right)$ be 
such that $t_{N}>\ldots>t_{1}>s>0$. Let us assume that $\rho_{m}$ is deterministic and let us take the conditional (to $s$ ) expectations in (13) for $t=t_{j}, j=1, \ldots N$. We then have for all $j=1, \ldots N$,

$$
\exp \left(-\rho_{m, t_{j}} t_{j}\right) M_{m, s}=\sum_{i} \lambda^{* i} \exp \left(-\rho^{i} t_{j}\right) M_{s}^{i}
$$

Let $\mathbf{X}(\omega)=\left(\lambda^{* 1} M_{s}^{1}(\omega),, \ldots, \lambda^{* N} M_{s}^{N}(\omega)\right.$ and $A=\left(\exp \left(-\rho^{i} t_{j}\right)\right)_{j, i}$. Then $A \mathbf{X}(\omega)=M_{m, s}(\omega) \mathbf{Y}$ with $\mathbf{Y}=\left(\exp \left(-\rho_{m, t_{1}} t_{1}\right), \ldots, \exp \left(-\rho_{m, t_{N}} t_{N}\right)\right)$. From Lemma 12 with $a_{i}=\exp \left(-\rho^{i}\right)$ and $b_{j}=t_{j}$, the matrix $\left(\exp \left(-\rho^{i} t_{j}\right)\right)_{i, j}$ is invertible and so is $A$ and we have $\mathbf{X}(\omega)=M_{m, s}(\omega) A^{-1} \mathbf{Y}$. Taking the expectation, we have $\Lambda=A^{-1} \mathbf{Y}$ with $\Lambda=\left(\lambda^{* 1}, \ldots \lambda^{* N}\right)$. Hence $\mathbf{X}(\omega)=M_{m, s}(\omega) \Lambda$ and therefore $M_{s}^{i}(\omega)=M_{m, s}(\omega)$ for all $i$ which contradicts our heterogeneous beliefs assumption.

\subsection{Appendix 2. Existence of a Production Equilib- rium and Related Results}

Existence of a production equilibrium is proven by a Negishi utility weights method. The proof of uniqueness extends Dana (1995) by introducing time, processes and a production set and is based on our assumption that shareholders have proportional endowments (no other endowments than their shares of the firm) and homogeneous utility indices (same CRRA instantaneous utility function for all shareholders).

\section{Concepts, notations and first results}

In this appendix, we assume that (P1), (P2) and (C) are fulfilled.

Let $Y_{+}=Y \cap X_{+}$and $Y_{\varsigma}=\left\{y \in Y_{+} \mid y \geq \varsigma\right\}$. From (P2), the sets $Y$ and $Y_{+}$and $Y_{\varsigma}$ are closed and convex and from (P1) (P2), $Y_{+}$and $Y_{\varsigma}$ are bounded, hence $\sigma\left(X, X^{\prime}\right)$ compact.

Definition 4 An allocation $\left(c^{1}, \ldots, c^{N}, y\right)$ is the specification of a consumption plan $c^{i} \in X_{+}$for each shareholder $i=1, \ldots, N$ and of a production plan $y \in Y_{+}$for the firm. The allocation is feasible if $\sum_{i} c^{i}=y$.

We first recall the following classical characterization of Pareto optima. 
Lemma 13 An allocation $\left(\bar{c}^{1}, \ldots, \bar{c}^{N}, \bar{y}\right)$ is a Pareto optimum if and only if there exists $\lambda \in \mathbb{R}_{+}^{N}$, such that $\left(\bar{c}^{1}, \ldots, \bar{c}^{N}\right)$ solves

$$
\max \left\{\sum \lambda^{i} U^{i}\left(c^{i}\right), c^{i} \geq 0 \text { for all } i, \sum c^{i}=y, y \in Y_{+}\right\}
$$

In order to compute explicitly Pareto optima, let us next introduce some notations. For $t \in \mathbb{T}, c \in \mathbb{R}_{+}$and $\lambda \in \mathbb{R}_{+}^{N}$, let $u(c, \lambda)$ and $\left(C^{i}(c, \lambda)\right)_{i}$ be defined by

$$
\begin{gathered}
u(t, c, \lambda)=\max \left\{\sum_{i} \lambda^{i} \exp \left(-\rho^{i} t\right) M_{t}^{i} u\left(c^{i}\right), c^{i} \geq 0, \forall i, \sum c^{i} \leq c\right\}, \\
C^{i}(t, c, \lambda)=\arg \max \left\{\sum_{i} \lambda^{i} \exp \left(-\rho^{i} t\right) M_{t}^{i} u\left(c^{i}\right), c^{i} \geq 0, \forall i, \sum c^{i} \leq c\right\} .
\end{gathered}
$$

We recall that the process $N(\lambda)$ is defined by

$$
N_{t}(\lambda)=\left(\sum_{i}\left(\lambda^{i} M_{t}^{i} \exp \left(-\rho^{i} t\right)\right)^{\frac{1}{1-\gamma}}\right)^{1-\gamma}
$$

One easily verifies that

$$
\begin{aligned}
u(c, \lambda) & =N(\lambda) u(c), \\
C^{i}(c, \lambda) & =S^{i}(\lambda) c
\end{aligned}
$$

where $S_{t}^{i}(\lambda)=\frac{\left(\lambda^{i} M_{t}^{i} e^{-\rho^{i} t}\right)^{\frac{1}{1-\gamma}}}{\sum_{j}\left(\lambda^{j} M_{t}^{j} e^{-\rho^{j} t}\right)^{\frac{1}{1-\gamma}}}$ represents shareholder's $i$ stochastic share of $c$. For any $(\omega, t, \lambda)$, the function $u(\omega, t, \cdot, \lambda)$ is differentiable with respect to $c$ on $] 0, \infty[$ and we have

$$
u_{c}(c, \lambda)=N(\lambda) u^{\prime}(c)
$$

From lemma 13 , the allocation $\left(\bar{c}^{1}, \ldots, \bar{c}^{I}, \bar{y}\right)$ is a Pareto optimum if and only if there exists $\lambda \in \mathbb{R}_{+}^{I}$, such that $\bar{y} \in Y_{+}$solves the representative shareholder problem $\mathcal{P}_{\lambda}$ :

$$
\max _{y \in Y_{+}} \sum_{t=0}^{T} E\left(u\left(t, y_{t}, \lambda\right)\right)=\max _{y \in Y_{+}} N(\lambda) \cdot u(y)
$$


and $\bar{c}_{t}^{i}=C^{i}(t, \bar{y}, \lambda)$ for all $i$. As $u(t, \cdot, \lambda)$ is increasing, the maximization may be restricted to $Y_{\varsigma}$. As $u(t, c, \cdot)$ is homogeneous of degree 1, $C^{i}(t, c, \cdot)$ is homogeneous of degree 0 for all $i$ and we may restrict attention to $\Lambda=\left\{\lambda \in \mathbb{R}_{+}^{I}: \sum_{i}\left(\lambda^{i}\right)^{\frac{1}{1-\gamma}}=1\right\}$.

For further use, let us introduce some notations.

Let $X_{M_{i}} \stackrel{\text { def }}{=}\left\{x: M_{i} x \in L^{1}\left(\Omega,\left(\mathcal{F}_{t}\right)_{t \in \mathbb{T}}, P\right)\right\}$ for all $i$ and $X_{M} \stackrel{\text { def }}{=} \cap_{i} X_{M_{i}}$. Let $X_{M}^{\prime}=\left\{M p, p \in L^{\infty}\left(\Omega,\left(\mathcal{F}_{t}\right)_{t \in \mathbb{T}}, P\right)\right\}$ with $M=\sum_{i} M_{i}$. For every $x \in X_{M}$ and $M p \in X_{M}^{\prime}$, let $x \cdot M p=: \sum_{t=0}^{T} E\left(M_{t} p_{t} x_{t}\right)=M p \cdot x$.

Let $L^{1}$ denote $L^{1}\left(\Omega,\left(\mathcal{F}_{t}\right)_{t \in \mathbb{T}}, P\right)$ and $L^{\infty}$ denote $L^{\infty}\left(\Omega,\left(\mathcal{F}_{t}\right)_{t \in \mathbb{T}}, P\right)$. We denote by $\rightarrow$ the convergence with respect to $\sigma\left(X, X^{\prime}\right)$ and by $\rightarrow_{M}$ the convergence with respect to the topology $\sigma\left(X_{M}, X_{M}^{\prime}\right)$. Note that $w_{n} \rightarrow_{M} w$ if and only if $M w_{n} \rightarrow M w$ with respect to the topology $\sigma\left(L^{1}, L^{\infty}\right)$. Let

$$
V=\left\{v=u(y): y \in Y_{\varsigma}\right\}
$$

By Assumptions (C) and (P1), for any $y \in Y_{\varsigma}$,

$$
|u(y)| \leq|u(\Xi)|+|u(\varsigma)| \stackrel{\text { def }}{=} A \in X_{M} .
$$

Therefore, $V \subset X_{M}$.

\section{Pareto Optima and Equilibria with transfers}

We start by proving a lemma which will be useful in many proofs.

Lemma 14 1. There exists $a>0$ such that $|N(\lambda)| \leq a M \in X_{M}^{\prime}$.

2. For $\left(\lambda_{n}\right) \subset \Lambda$ such that $\lambda_{n} \rightarrow \lambda^{\prime}$, we have $N\left(\lambda_{n}\right) \rightarrow N\left(\lambda^{\prime}\right)$ for $\sigma\left(X_{M}^{\prime}, X_{M}\right)$.

3. If $\left(y_{n}\right) \subset Y_{\varsigma}$ and $y_{n} \rightarrow_{\text {a.e. }} y^{\prime}$, then $u\left(y_{n}\right) \rightarrow_{M} u\left(y^{\prime}\right)$. Let $\left(v_{n}\right) \subset V$ $v_{n} \rightarrow$ a.e. $v^{\prime}$ and $\lambda_{n} \rightarrow \lambda^{\prime}$, then $N\left(\lambda_{n}\right) v_{n} \rightarrow_{L_{1}} N\left(\lambda^{\prime}\right) v^{\prime}$.

Proof. 1. $b \rightarrow\left(\sum_{i}\left|b_{i}\right|^{\frac{1}{1-\gamma}}\right)^{1-\gamma}$ is bounded over the simplex of $\mathbb{R}^{n}$, hence there exists $a>0$ such that $\left(\sum_{i}\left|b_{i}\right|^{\frac{1}{1-\gamma}}\right)^{1-\gamma} \leq a \sum_{i}\left|b_{i}\right|$. We then have $N_{t}(\lambda) \leq a\left(\sum_{i}\left(\lambda^{i} M_{t}^{i} \exp \left(-\rho^{i} t\right)\right)\right) \leq a M_{t}$ and $N(\lambda) \in X_{M}^{\prime}$. 
2. For $\lambda_{n} \rightarrow \lambda^{\prime}$ and $w \in X_{M}$ we have $w N\left(\lambda_{n}\right) \rightarrow$ a.e. $w N\left(\lambda^{\prime}\right)$ and $\left|w N\left(\lambda_{n}\right)\right| \leq a M|w| \in L^{1}$. By Lebesgue Theorem, we then have $w N\left(\lambda_{n}\right) \rightarrow{ }^{1}$ $w N\left(\lambda^{\prime}\right)$ for all $w \in X_{M}$.

3. If $y_{n} \rightarrow_{a . e . ~} y^{\prime}$, for $x \in X_{M}^{\prime}, x u\left(y_{n}\right) \rightarrow_{\text {a.e. }} x u\left(y^{\prime}\right)$ and $\left|x u\left(y_{n}\right)\right| \leq$ $|x A| \in L^{1}$. By Lebesgue Theorem, we have $u\left(y_{n}\right) \rightarrow_{M} u\left(y^{\prime}\right)$. If $\lambda_{n} \rightarrow \lambda^{\prime}$ and $v_{n} \rightarrow_{\text {a.e. }} v^{\prime}, N\left(\lambda_{n}\right) v_{n} \rightarrow_{\text {a.e. }} N\left(\lambda^{\prime}\right) v^{\prime}$ and $\left|N\left(\lambda_{n}\right) v_{n}\right| \leq a M A \in L^{1}$ and we conclude similarly.

We now characterize the Pareto optima. To this end, let us reconsider problem $\mathcal{P}_{\lambda}$ and introduce a notation:

$$
\max _{y \in Y_{\varsigma}} \sum_{t=0}^{T} E\left[u\left(t, y_{t}, \lambda\right)\right]=\max _{v \in V} F(v, \lambda) \text { with } F(v, \lambda)=N(\lambda) \cdot v \text {. }
$$

From Lemma 14, problem $\mathcal{P}_{\lambda}$ is well defined. Let us show that it admits a unique solution $y_{\lambda}$ ( or $v_{\lambda}=u\left(y_{\lambda}\right)$ depending on the chosen formulation).

Lemma 15 The problem $\mathcal{P}_{\lambda}$ has a unique solution denoted $y_{\lambda}$. Therefore $F(., \lambda)$ reaches its maximum on $V$ at a unique point $v_{\lambda}$.

Proof. Let $\left(y_{n}\right) \subset Y_{\varsigma}$ and $\varepsilon>0$ be such that $\left\|y_{n}-y^{\prime}\right\|_{r} \rightarrow 0$ and $N(\lambda) \cdot u\left(y_{n}\right)>N(\lambda) \cdot u\left(y^{\prime}\right)+\varepsilon$ for all $n$. There exists a subsequence $y_{\varphi(n)} \rightarrow$ a.e. $y^{\prime}$ and, by Lemma 14 , we have $N(\lambda) u\left(y_{\varphi(n)}\right) \rightarrow_{L_{1}} N(\lambda) u\left(y^{\prime}\right)$ which contradicts our assumption. Hence $y \rightarrow N(\lambda) \cdot u(y)$ is strongly u.s.c. and therefore weakly u.s.c. on $Y_{\varsigma}$ which is weakly compact. Therefore it admits a maximum $y_{\lambda}$. Uniqueness of $y_{\lambda}$ follows from the strict concavity of $u$, that of $v_{\lambda}$ from the monotonicity of $u$.

From Lemmas 13 and 15 and Equation (22), Pareto optima may be described as follows:

Lemma 16 An allocation $\left(\bar{c}^{1}, \ldots, \bar{c}^{N}, \bar{y}\right)$ is a Pareto optimum if and only if there exists $\lambda \in \mathbb{R}_{+}^{N}$, such that $\bar{y}=y_{\lambda}$ and $\bar{c}^{i}=C^{i}\left(y_{\lambda}, \lambda\right)$ for every $i$.

Let us next recall the definition of an equilibrium with transfers.

Definition 5 An allocation $\left(\bar{c}^{1}, \ldots, \bar{c}^{N}, \bar{y}\right)$ with a strictly positive price process $\bar{p}$ is an equilibrium with transfers if it verifies: 
1. $\bar{y}$ maximizes profit that is $: \bar{p} \cdot y \leq \bar{p} \cdot \bar{y}$ for all $y \in Y$,

2. For every $i, \bar{c}^{i}$ maximizes $U^{i}(c)$ subject to $\bar{p} \cdot c \leq \bar{p} \cdot \bar{c}^{i}$.

We now prove a second welfare theorem:

Lemma 17 For any $\lambda \in \mathbb{R}_{+}^{N}$, the allocation $\left(\left(C^{i}\left(y_{\lambda}, \lambda\right)\right)_{i}, y_{\lambda}\right)$ with the price $u_{c}\left(y_{\lambda}, \lambda\right)$ is an equilibrium with transfer.

Proof. Let us first verify that the price process is in $X^{\prime}$. From (23), Lemma 14 and Assumption (C), we have

$$
u_{c}\left(y_{\lambda}, \lambda\right)=N(\lambda) u^{\prime}\left(y_{\lambda}\right) \leq a M u^{\prime}\left(y_{\lambda}\right) \leq a M \varsigma^{\gamma-1} \in X^{\prime}
$$

As $y_{\lambda}$ solves $\mathcal{P}_{\lambda}, u_{c}\left(y_{\lambda}, \lambda\right) \in N_{Y}\left(y_{\lambda}\right)$. Therefore $y_{\lambda}$ maximizes profit at price $u_{c}\left(y_{\lambda}, \lambda\right)$ proving the first property of an equilibrium with transfers. The remainder of the proof which is totally standard is skipped.

\section{A useful continuity property}

The aim of this subsection is to prove that if $\lambda_{n} \rightarrow \lambda$, then $v_{\lambda_{n}} \rightarrow_{M} v_{\lambda}$ where we recall that :

$$
F\left(v_{\lambda}, \lambda\right)>F(v, \lambda), \forall v \in V, \lambda \in \Lambda
$$

\section{Lemma 18 1. $V$ and $M V$ are convex,}

2. $M V$ is sequentially $\sigma\left(L^{1}, L^{\infty}\right)$ compact and $V$ is $\sigma\left(X_{M}, X_{M}^{\prime}\right)$ sequentially compact.

Proof. To prove assertion 1 , note first that if the $\mathcal{F}$-adapted process $t$ is such that $u(\varsigma) \leq t \leq u(y)$ for some $y \in Y_{\varsigma}$, then $t \in V$. Indeed the process $u^{-1}(t)$ is $\mathcal{F}$-adapted and $\varsigma \leq u^{-1}(t) \leq y$. Therefore $u^{-1}(t) \in Y_{\varsigma}$ and $t=u\left(u^{-1}(t)\right) \in V$. To show the convexity of $V$, let $y_{i} \in Y_{\varsigma}$ for $i=1,2$ and $0 \leq \lambda \leq 1$. As $u\left(y_{i}\right) \geq u(\varsigma)$ for $i=1,2$ and from the concavity of $u$

$$
u(\varsigma) \leq \lambda u\left(y_{1}\right)+(1-\lambda) u\left(y_{2}\right) \leq u\left(\lambda y_{1}+(1-\lambda) y_{2}\right) .
$$

From our previous remark $\lambda u\left(y_{1}\right)+(1-\lambda) u\left(y_{2}\right) \in V$ which implies that $V$ is convex and therefore $M V$ is convex. To prove assertion 2 , let us first 
prove that $M V$ is closed. Let $M u\left(y_{n}\right) \rightarrow z$ in the norm topology of $L^{1}$ with $y_{n} \in Y_{\varsigma}$. Then some subsequence $M u\left(y_{n_{k}}\right) \rightarrow z$ a.e. Hence $y_{n_{k}} \rightarrow u^{-1}\left(\frac{z}{M}\right)$ a.e. and as $y_{n} \leq \Xi$ for any $n$, the convergence is also in $L^{r}$. As $Y_{\varsigma}$ is closed, there exists $y \in Y_{\varsigma}$ such that $y=u^{-1}\left(\frac{z}{M}\right)$ and $z=M u(y) \in V$. Hence $M V$ is norm closed and as it is convex, it is $\sigma\left(L^{1},{ }^{\infty}\right)$ closed. From (24), $M V$ is equi-integrable. It then follows from Dunford-Pettis' theorem that it is sequentially $\sigma\left(L^{1}, L^{\infty}\right)$ compact. Hence every sequence $M v_{n}$ has a subsequence converging weakly in $M V$ or equivalently every sequence $v_{n}$ has a subsequence converging in $V$ in the $\sigma\left(X_{M}, X_{M}^{\prime}\right)$ topology proving that $V$ is $\sigma\left(X_{M}, X_{M}^{\prime}\right)$ sequentially compact.

We now prove the main result of this subsection.

Lemma 19 1. If $v_{n} \rightarrow_{M} v^{\prime}$ and $\lambda_{n} \rightarrow \lambda^{\prime}$, then $F\left(v_{n}, \lambda_{n}\right) \rightarrow F\left(v^{\prime}, \lambda^{\prime}\right)$.

2. If $\lambda_{n} \rightarrow \lambda$, then $v_{\lambda_{n}} \rightarrow_{M} v_{\lambda}$.

Proof. To prove assertion 1, we have

$$
\left|F\left(v^{\prime}, \lambda\right)-F\left(v_{n}, \lambda_{n}\right)\right| \leq\left|F\left(v^{\prime}, \lambda^{\prime}\right)-F\left(v_{n}, \lambda^{\prime}\right)\right|+\left|F\left(v_{n}, \lambda_{n}\right)-F\left(v_{n}, \lambda^{\prime}\right)\right| .
$$

$\left|F\left(v^{\prime}, \lambda^{\prime}\right)-F\left(v_{n}, \lambda^{\prime}\right)\right|=\left|N\left(\lambda^{\prime}\right) \cdot\left(v^{\prime}-v_{n}\right)\right| \rightarrow 0$ as $v_{n} \rightarrow_{M} v^{\prime}$ and $N\left(\lambda^{\prime}\right) \in$ $X_{M}^{\prime}$. Moreover,

$\left|F\left(v_{n}, \lambda_{n}\right)-F\left(v_{n}, \lambda^{\prime}\right)\right|=\left|\left(N\left(\lambda_{n}\right)-N\left(\lambda^{\prime}\right)\right) \cdot v_{n}\right| \leq\left|\left(N\left(\lambda_{n}\right)-N\left(\lambda^{\prime}\right)\right)\right| \cdot A$.

and from Lemma $14\left|F\left(v_{n}, \lambda_{n}\right)-F\left(v_{n}, \lambda^{\prime}\right)\right| \rightarrow 0$.

To prove assertion 2 , let $\lambda_{n} \rightarrow \lambda$, then from Lemma 18 assertion 2 , $v_{\lambda_{n}}$ has a subsequence, still denoted $v_{\lambda_{n}}$, such that $v_{\lambda_{n}} \rightarrow_{M} \bar{v}$ with $\bar{v} \in V$. From (25), for every $n$, we have $F\left(v_{\lambda_{n}}, \lambda_{n}\right)>F\left(v, \lambda_{n}\right), \forall v \in V$. Taking the limit as $n \rightarrow \infty$, we obtain from assertion $1 F(\bar{v}, \lambda) \geq F(v, \lambda), \forall v \in V$. As $F(\cdot, \lambda)$ has as unique maximizer $v_{\lambda}, \bar{v}=v_{\lambda}$. Hence the sequence $v_{\lambda_{n}}$ has as unique cluster point $v_{\lambda}$ and therefore $v_{\lambda_{n}} \vec{M}_{M} v_{\lambda}$. 


\section{Definition and Properties of the transfer map}

We may now define the transfer map $\mathcal{T}$ by

$$
\mathcal{T}^{i}(\lambda)=u_{c}\left(y_{\lambda}, \lambda\right) \cdot\left(C^{i}\left(y_{\lambda}, \lambda\right)-\nu^{i} y_{\lambda}\right)=G^{i}\left(v_{\lambda}, \lambda\right), \text { for } i=1, \ldots, N
$$

with $G^{i}(v, \lambda)=\gamma N(\lambda) \cdot\left(S^{i}(\lambda)-\nu^{i}\right) v$. As $u_{c}\left(y_{\lambda}, \lambda\right) \in X^{\prime}$ for every $\lambda, \mathcal{T}$ is well-defined. Let us quote a continuity property of $G$ whose proof, similar to that of the continuity of $F$ is omitted.

Lemma 20 If $\left(v_{n}, \lambda_{n}\right) \subset V \times \Lambda$ is such that $v_{n} \rightarrow_{M} v^{\prime}$ and $\lambda_{n} \rightarrow \lambda^{\prime}$, then $G\left(v_{n}, \lambda_{n}\right) \rightarrow G\left(v^{\prime}, \lambda^{\prime}\right)$.

Proposition $21 \mathcal{T}$ is continuous on $\Lambda$ and there exists $\lambda^{*}$ with $\lambda^{* i}>0$ for all $i$ such that $\mathcal{T}^{i}\left(\lambda^{*}\right)=0$ for all $i$.

Proof. To show the continuity of $\mathcal{T}^{i}$ on $\Lambda$, let $\lambda_{n} \rightarrow \lambda^{\prime}$. From Lemma 19, $v_{\lambda_{n}} \rightarrow_{M} v\left(\lambda^{\prime}\right)$. Hence from Lemma $20, G^{i}\left(v_{\lambda_{n}}, \lambda_{n}\right) \rightarrow G^{i}\left(v_{\lambda^{\prime}}, \lambda^{\prime}\right)$ proving the continuity of $\mathcal{T}^{i}$. The proof of existence of a $\lambda^{*}$ with $\lambda^{* i}>0$ for all $i$ such that $\mathcal{T}^{i}\left(\lambda^{*}\right)=0$ for all $i$ is standard and follows the properties of $T$ (continuity, boundary behavior $\mathcal{T}^{i}(\lambda)<0$ when $\lambda^{i}=0$ for some $i$, and $\sum \mathcal{T}^{i}(\lambda)=0$ for every $\left.\lambda \in \Lambda\right)$.

Proof of Theorem 4, Assertion 1. $\mathcal{T}$ has a zero to which corresponds a Pareto optimum with zero transfer payments. It is therefore a production equilibrium. Let us show that it is unique. Assume that there are two equilibria $\left(\left(\hat{c}^{1}, \ldots, \hat{c}^{N}\right), \hat{y}, \hat{p}\right)$ and $\left(\left(\widetilde{c}^{1}, \ldots, \widetilde{c}^{N}\right), \widetilde{y}, \widetilde{p}\right)$ where without loss of generality, prices are such that $\hat{p} \cdot \hat{y}=\widetilde{p} \cdot \widetilde{y}=1$. Then $\hat{c}^{i}$ is the optimal solution to the problem

$$
\max U^{i}(c) \text { s.t. } \hat{p} \cdot c \leq \nu^{i}
$$

and $\tilde{c}^{i}$ is the optimal solution to the problem

$$
\max U^{i}(c) \text { s.t. } \tilde{p} \cdot c \leq \nu^{i}
$$

From Dana (1995) Proposition 2.1, as utilities are homogeneous, we have for every $i$,

$$
(\hat{p}-\tilde{p}) \cdot\left(\hat{c^{i}}-\tilde{c^{i}}\right)<0
$$


Summing over $i$, we obtain

$$
(\hat{p}-\widetilde{p}) \cdot(\hat{y}-\widetilde{y})<0
$$

From the definition of a production equilibrium, we have $\hat{p} \cdot \hat{y} \geq \hat{p} \cdot \widetilde{y}$ and $\widetilde{p} \cdot \widetilde{y} \geq \widetilde{p} \cdot \hat{y}$ which leads to a contradiction. The uniqueness of the vector of equilibrium utility weights follows immediately.

Remark 22 Existence and uniqueness of an exchange equilibrium for a given $y \in X_{+}$follows from the previous theorem by choosing $Y=\{y\}-X_{+}$. 